\title{
Magnétisme de bande et Supraconductivité : l'apport de la diffusion inélastique des neutrons
}

\author{
Yvan Sidis \\ Laboratoire Léon Brillouin, CEA-CNRS, CE-Saclay, 91191 Gif-sur-Yvette, France
}

\begin{abstract}
Résumé. La technique de diffusion des neutrons permet d'étudier les corrélations magnétiques statiques et dynamiques sur une large gamme d'énergies et de vecteurs d'onde dans l'espace des phases. Elle offre une mesure directe de la partie imaginaire de la susceptibilité magnétique généralisé qui contient toutes les informations relatives aux excitations magnétiques d'un système. Dans un métal, les propriétés magnétiques sont principalement véhiculés par les spins des électrons itinérants. Nous décrirons comment la susceptibilité magnétiques est déterminé à partir des propriétés d'un liquide d'électrons, puis comment les excitations magnétiques rétro-agissent sur les propriétés des électrons. En passant d'un état métallique à un état supraconducteur, les électrons s'apparient pour former des paires d'électrons condensés dans un état quantique macroscopique. Aux électrons, se substituent de nouvelles quasiparticules. Nous décrirons comment la susceptibilité magnétique est modifiée et pour quelle raison de nouvelles excitations collectives peuvent apparaitre. Nous insisterons sur le fait que les électrons engendrent des excitations magnétiques qu'ils peuvent ensuite absorber, émettre ou encore (théoriquement) s'échanger pour induire une interaction attractive effective conduisant à la formation de paires d'électrons supraconductrices. Une fois ces concepts présentés, nous donnerons quelques exemples de la mesure de la partie imaginaire de la susceptibilité magnétique par diffusion inélastique des neutrons dans un métal normal, puis dans des matériaux supraconducteurs. Nous conclurons par quelques remarques d'ordre général permettant au lecteur de s'ouvrir à des problématiques que nous n'aurons pas abordés.
\end{abstract}

\section{TECHNIQUE DE DIFFUSION INÉLASTIQUE DES NEUTRONS}

La section de diffusion inélastique magnétique [1] s'exprime en fonction de la transformée de Fourier de la fonction de corrélation spin-spin $S_{\alpha, \beta}(\mathbf{Q}, \omega)$ (les indices $\alpha, \beta$ correspondent aux coordonnées cartésiennes $\mathrm{x}, \mathrm{y}, \mathrm{z})$ :

$$
\frac{d^{2} \sigma}{d \Omega d \omega}=\frac{k_{f}}{k_{i}} r_{0}^{2} F^{2}(Q) \sum_{\alpha, \beta}\left(\delta_{\alpha, \beta}-\frac{Q_{\alpha} Q_{\beta}}{|Q|^{2}}\right) S_{\alpha, \beta}(\mathbf{Q}, \omega)
$$

où $k_{i}$ et $k_{f}$ sont les vecteurs d'onde incident et diffusé des neutrons, $r_{0}^{2}=0.292$ barns, $F(Q)$ représente le facteur de forme magnétique. Le vecteur d'onde transféré $\mathbf{Q}$ est donné par la combinaison de $\mathbf{q}$ un vecteur d'onde de la première zone de Brillouin et $\mathbf{G}$ un vecteur d'onde du réseau réciproque, tel que: $\mathbf{Q}=\mathbf{q}+\mathbf{G}$. Toutes les coordonnées dans l'espace réciproque $\left(Q_{x}, Q_{y}, Q_{z}\right)($ ou $(H, K, L))$ sont 


\section{Collection SFN}

habituellement donnés en unités réduites. Par exemple, pour un système tétragonal dont les paramètres du réseau cristallin sont $a=b$ et $c$, elles seront exprimées en unité $(2 \pi / a, 2 \pi / b, 2 \pi / c)$.

En accord avec le théorème de fluctuation-dissipation, la fonction de corrélation spin-spin est directement proportionnelle à la partie imaginaire de la susceptibilité magnétique généralisée, pondérée par le facteur de balance détaillé :

$$
S_{\alpha \beta}(\mathbf{Q}, \omega)=\frac{1}{\pi} \frac{1}{1-\exp \left(-\hbar \omega / k_{B} T\right)} \frac{\operatorname{Im} \chi_{\alpha \beta}(\mathbf{Q}, \omega)}{\left(g \mu_{B}\right)^{2}} .
$$

La mesure de diffusion inélastique des neutrons permet en principe d'obtenir une détermination complète de la susceptibilité magnétique. Elle donne un accès direct à $\operatorname{Im} \chi_{\alpha \beta}(\mathbf{Q}, \omega)$ qui est une fonction impaire de $\omega$. Cette fonction est reliée par la relation de Kramers-Kronig à $\operatorname{Re} \chi_{\alpha \beta}(\mathbf{Q}, \omega)$ qui est une fonction impaire de $\omega$.

$$
\begin{aligned}
& \operatorname{Re} \chi_{\alpha \beta}(\mathbf{q}, \omega)=\frac{1}{\pi} \mathcal{P} \int_{-\infty}^{\infty} d \omega^{\prime} \frac{\operatorname{Im} \chi_{\alpha \beta}\left(\mathbf{q}, \omega^{\prime}\right)}{\omega-\omega^{\prime}} \\
& \operatorname{Im} \chi_{\alpha \beta}(\mathbf{q}, \omega)=-\frac{1}{\pi} \mathcal{P} \int_{-\infty}^{\infty} d \omega^{\prime} \frac{\operatorname{Re} \chi_{\alpha \beta}\left(\mathbf{q}, \omega^{\prime}\right)}{\omega-\omega^{\prime}}
\end{aligned}
$$

$\mathcal{P}$ donne la valeur principale de la fonction qui suit. On mesure donc la partie imaginaire de la susceptibilité magnétique, à partir de laquelle on peut déterminer la partie réelle et ainsi obtenir une description complète de la susceptibilité magnétique.

De façon générale, seuls les termes diagonaux du tenseur de susceptibilité sont non nuls $(\alpha=\beta)$ et pour un système paramagnétique, $\chi_{x x}, \chi_{y y}$ et $\chi_{z z}$ sont identiques, tout du moins en l'absence d'anisotropie de spin.

En réalité, l'expérimentateur se heurte en pratique à deux problèmes. Les mesures doivent être déconvoluées de la fonction de résolution instrumentale. Puis, la gamme d'énergies accessibles par diffusion inélastique des neutrons est limitée, la transformation de Kramers-Kronig qui donne $\operatorname{Re} \chi_{\alpha \beta}(\mathbf{Q}, \omega)$ se fait donc jusqu'à une énergie seuil.

\section{- susceptibilité généralisé :}

La susceptibilité généralisé [2] permet d'estimer la valeur moyenne d'une observable $<B>$ sous l'action d'un champ $a<\omega>$ engendré par l'observable conjugée A, tel que $<B>=\chi_{B A} a(\omega)$, avec :

$$
\chi_{B A}(\omega)=\sum_{n, p} \frac{\Pi_{n}-\Pi_{p}}{E_{n}-E_{p}-\omega-i \epsilon}<n|B| p><p|A| n>
$$

avec $\Pi_{n}=\frac{e^{-E_{n} / k_{B} T}}{Z}$ où $\mathrm{Z}$ représente la fonction de partition. $|n\rangle$ correspond à un état du système d'énergie $E_{n}$.

Pour la susceptibilité magnétique longitudinale $\chi_{z z}: A=g \mu_{B} S_{q}^{z}$ et $B=g \mu_{B} S_{-q}^{z}$. Pour la susceptibilité magnétique transverse $\chi_{+-}: A=g \mu_{B} S_{q}^{+}$et $B=g \mu_{B} S_{-q}^{-}$, avec $S_{q}^{+,-}=S_{q}^{x} \pm i S_{q}^{y}$. Notons que pour un système paramagnétique, $\chi_{+-}=2 \chi_{z z}$.

\section{PROPRIÉTÉS MAGNÉTIQUES D’UN MÉTAL NORMAL}

\subsection{Electrons sans interaction}

Dans un métal [3], les électrons qui portent un spin $\frac{1}{2}$ sont délocalisés. Leurs propriétés sont décrites dans l'espace des phases. Chaque électron occupe un état de Bloch $|\mathbf{k}, \sigma\rangle$. La distribution de ces états se présente sous la forme d'une bande, caractérisée par une relation de dispersion en énergie 

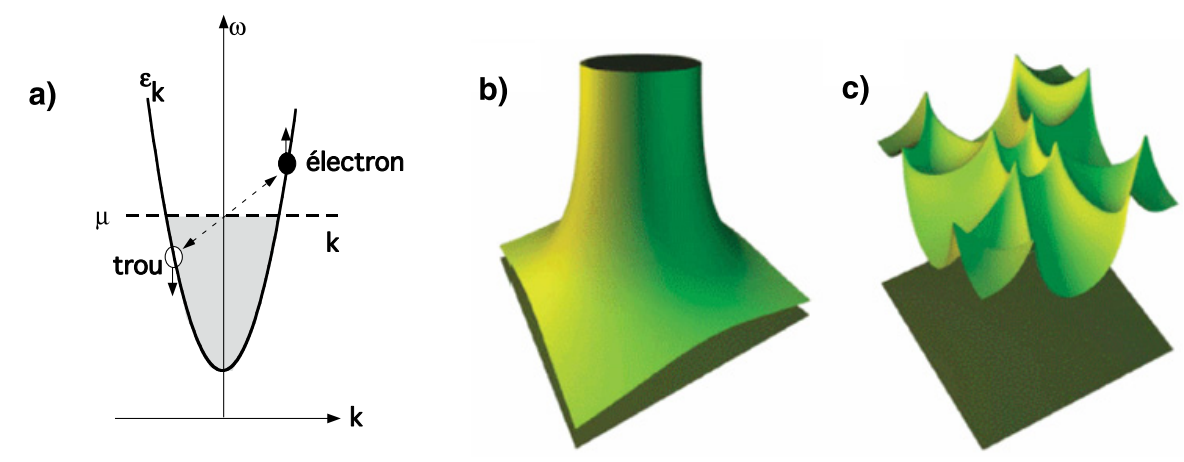

Figure 1. a) Description schématique d'une excitation électron-trou avec reversement du spin. Susceptibilité statique : b) par un gaz d'électron (modèle du jellium), c) pour des électrons se déplaçant sur un réseau plan carré [6].

$\epsilon_{k, \sigma}$. Les électrons sont des fermions et à température nulle, ils occupent tous les états de plus basse énergie du système en respectant le principe de Pauli (un même état électronique peut au plus être occupé par deux électrons de spins $\sigma$ et $-\sigma$ ). Tous les états sont alors occupés jusqu'à une énergie seuil, l'énergie de Fermi qui correspond à l'énergie du potentiel chimique $\mu$ (Fig. 1a). Pour une température quelconque T, l'état d'occupation de chaque état est donné par la fonction de Fermi-Dirac : $n^{F}\left(\xi_{k, \sigma}\right)=\left[\exp \left(\left(\xi_{k, \sigma} / k_{B} T\right)+1\right)\right]^{-1}$, où $\xi_{k, \sigma}$ représente la relation de dispersion en fonction du niveau de Fermi, soit $\xi_{k, \sigma}=\epsilon_{k, \sigma}-\mu$.

Le Hamiltonien n'a pas d'autre terme que l'énergie cinétique et s'écrit :

$$
H=\sum_{k, \sigma} \xi_{k, \sigma} c_{k, \sigma}^{+} c_{k, \sigma}
$$

Ici, les opérateurs de création $c_{k, \sigma}^{+}$et d'annihilation $c_{k, \sigma}$ permettent respectivement d'ajouter ou d'enlever un électron dans un état $|\mathbf{k}, \sigma\rangle$. Lorsque l'on enlève un électron, on crée une lacune électronique que l'on appelle un trou. Ce trou se comporte comme un anti-électron de charge $(+e)$ et d'énergie $\left(-\xi_{k}\right)$ opposées.

Pour décrire la susceptibilité magnétique d'un tel système, nous considérons la transformée de Fourier de l'aimantion $M_{q}=g \mu_{B} S_{q}$ qui est reliée à une modulation de la densité de spin. $g(=2)$ est le facteur de Landé et $\mu_{B}$ le magnéton de Bohr. L'opérateur densité de spin $S_{q}$ s'exprime comme une combinaison linéaire de paires électron-trou et est défini comme suit :

$$
\begin{array}{r}
S_{q}=\frac{1}{N} \sum_{k, \sigma, \sigma^{\prime}} c_{k+q, \sigma}^{+} \bar{\sigma} c_{k, \sigma^{\prime}} \\
\left\{\begin{array}{l}
S_{q}^{z}=\frac{1}{N} \frac{1}{2} \sum_{k} c_{k+q, \uparrow}^{+} c_{k, \uparrow}-c_{k+q, \downarrow}^{+} c_{k, \downarrow} \\
S_{q}^{+}=S_{q}^{x}+i S_{q}^{y}=\frac{1}{N} \sum_{k} c_{k+q, \uparrow}^{+} c_{k, \downarrow} \\
S_{q}^{-}=S_{q}^{x}+i S_{q}^{y}=\frac{1}{N} \sum_{k} c_{k+q, \downarrow}^{+} c_{k, \uparrow}
\end{array}\right\}
\end{array}
$$

où $\bar{\sigma}$ représente les matrices de Pauli.

En utilisant l'expression de la susceptibilité généralisé (Eq. (1.4)), on obtient la susceptibilité magnétique d'un système d'électrons sans interaction. Celle-ci s'écrit en fonction de la susceptibilité 


\section{Collection SFN}

de Lindhard sans dimension $\chi^{o}(q, \omega)[1,3,4]$, tel que :

$$
\begin{aligned}
\chi_{\sigma, \sigma^{\prime}}^{o}(q, \omega) & =-\frac{1}{N} \sum_{k} \frac{n^{F}\left(\xi_{k \sigma^{\prime}}\right)-n^{F}\left(\xi_{k+q \sigma}\right)}{\omega+\xi_{k \sigma^{\prime}}-\xi_{k+q, \sigma \sigma}+i \epsilon} \\
\chi_{z z}(q, \omega) & =\frac{1}{4}\left(g \mu_{B}\right)^{2}\left(\chi_{\uparrow \uparrow}^{o}(q, \omega)+\chi_{\downarrow \downarrow}^{o}(q, \omega)\right) \\
\chi_{+-}(q, \omega) & =\left(g \mu_{B}\right)^{2} \chi_{\uparrow \downarrow}^{o}(q, \omega) .
\end{aligned}
$$

Cette fonction décrit toutes les excitations électron-trou autorisées dans le système (Fig. 1a). L'ensemble de ces excitations élémentaires forme un continuum (continuum de Stoner). La structure de ce continuum est conditionnée par la relation de dispersion des électrons. A titre d'exemple, la Figure $1 \mathrm{~b}$ montre la structure de la partie réelle de la susceptibilité à énergie nulle $\operatorname{Re} \chi^{o}(q, 0)$ pour un gaz d'électrons, avec comme relation de dispersion $\xi_{k}=\frac{\hbar^{2}}{2 m}|k|^{2}-\mu$. $\operatorname{Re} \chi^{o}(q, 0)$ est dominé pas les processus à $2 k_{F}$, qui font passer les électrons d'un état $\left|-k_{F}\right\rangle$ au niveau de Fermi à un état $\left|k_{F}\right\rangle$ au niveau de Fermi. La Figure $1 \mathrm{c}$ montre $\operatorname{Re} \chi^{o}(q, 0)$ pour des électrons se déplaçant sur un réseau plan carré sautant de site à site, tel que $\xi_{k}=-2 t\left(\cos \left(k_{x}\right)+\cos \left(k_{y}\right)\right)-\mu$. $\operatorname{Re} \chi^{o}(q, 0)$ est beaucoup plus structuré dans ce cas, ce qui illustre l'importance du réseau cristallin dans lequel les électrons évoluent.

\subsection{Electrons corrélés}

Nous considérons à présent le cas d'un système d'électrons en interaction. Nous prenons pour l'illustrer le cas simple du modèle de Hubbard. Dans ce modèle, on s'intéresse à l'effet de la répulsion Coulombienne intra-site qui limite (ou interdit) la double occupation d'un même site par deux électrons de spins opposés. Dans l'espace réel, le Hamiltonien de Hubbard s'écrit : $H=H_{o}+H^{\prime}$. $H_{o}$ représente le Hamiltonien sans interaction et ne contient que l'énergie cinétique. $H^{\prime}=U \sum_{i} n_{i, \uparrow} n_{i, \downarrow}$ correspond au terme d'interaction. $U$ est l'énergie de répulsion Coulombien intra-site. $n_{i, \sigma}=c_{i, \sigma}^{+} c_{i, \sigma}$ indique la probabilité d'occupation du site i par un électron de spin $\sigma$. Dans l'espace des phases, $H^{\prime}$ s'écrit :

$$
H^{\prime}=\frac{U}{N} \sum_{k, k^{\prime}, q} c_{k+q \uparrow}^{+} c_{k \uparrow} c_{k^{\prime}-q \downarrow}^{+} c_{k^{\prime} \downarrow} .
$$

Dans l'approximation de la phase aléatoire (RPA) [4, 5], la susceptibilité de spin $\chi^{R P A}$ s'exprime en fonction de la susceptibilité sans interaction $\chi^{o}$ et de la répulsion coulombienne intra-site :

$$
\chi^{R P A}(q, \omega)=\frac{\chi^{o}(q, \omega)}{1-U \chi^{o}(q, \omega)} .
$$

Pour en comprendre la signification, on peut remettre cette expression sous la forme :

$$
\chi^{R P A}(q, \omega)=\chi^{o}(q, \omega)\left(1+U \chi^{o}(q, \omega)\right) .
$$

Puis en présence d'un champ magnétique extérieur $H_{-q}^{z}$, l'aimantation $M_{q}^{z}$ prend la forme:

$$
M_{q}^{z}=\chi_{z z}^{R P A}(q) H_{-q}^{z}=\chi_{z z}^{o}(q)\left(H_{-q}^{z}+\frac{2 U}{\left(g \mu_{B}\right)^{2}} M_{q}^{z}\right) .
$$

En présence d'un champ magnétique extérieur, le système d'électrons corrélés est décrit comme un système d'électrons indépendants en présence d'un champ magnétique extérieur auquel s'ajoute un champ moléculaire résultant des corrélations électroniques.

L'effet de la répulsion électronique entre électrons est donc d'amplifier la susceptibilité magnétique sans interaction. Le facteur $\left[1-U \chi^{o}(q, \omega)\right]^{-1}$ est le facteur d'amplification de Stoner. Une transition magnétique se produit lorsque le critère de $\operatorname{Stoner}\left(1-U \operatorname{Re} \chi^{o}(q, 0)=0\right)$ est réalisé. Dans ce cas, la 
susceptibilité magnétique statique diverge et le système devient magnétiquement instable. Le système transite vers une phase magnétique ordonnée (phase ferromagnétique ou phase onde de densité de spins).

La susceptibilité RPA permet de sentir l'effet des interactions, mais elle n'est justifiée que lorsque les effets des corrélations sont modérés, c'est-à-dire lorsque $U$ est plus faible que la largeur de la bande électronique. Dans ce cas l'effet des corrélations peut être traité en perturbation. De même, lorsque le système est proche d'une instabilité magnétique, la susceptibilité RPA donne au mieux une description très qualitative de la susceptibilité magnétique du système.

\subsection{Forme approchée de la susceptibilité magnétique}

Lorsque le système approche d'une instabilité magnétique, le poids spectral des fluctuations magnétiques est progressivement transféré vers les basses énergies et se structure autour du vecteur d'onde $\mathbf{Q}$ qui est caractéristique de l'instabilité magnétique. On peut procéder à un développement limité de la susceptibilité magnétique dans la limite $\omega \rightarrow 0$ et $(q-Q) \rightarrow 0$ (développement de OrsteinZernike) [4] :

$$
\chi(q, \omega)=\frac{\chi(Q)}{1+\xi^{2}(q-Q)^{2}-i \omega / \omega_{s f}} .
$$

Cette expression permet d'estimer l'ordre de grandeur de la partie réelle de la susceptibilité magnétique $\chi(Q)$, des longueurs de corrélation magnétiques $\xi$ et de l'énergie caractéristique des fluctuations magnétiques $\omega_{s f}$. Cette expression approchée de la susceptibilité magnétique est très générale et n'est pas spécifique à un système d'électrons itinérants. Par ailleurs, cette expression doit être modifiée dans le cas d'une instabilité ferromagnétique $(Q=0)$ en remplaçant $\omega$ par $\frac{\omega}{q}$.

\subsection{Spectre d'excitations des fermions}

Nous avons vu que la susceptibilité magnétique dans un métal faisait intervenir des processus d'excitations électron-trou, c'est-à-dire des processus à deux particules. Sous l'effet des corrélations électroniques, la susceptibilité magnétique peut également être amplifiée. De même, les propriétés des électrons, qui interagissent avec les fluctuations magnétiques vont être modifiées [5, 7]. Les propriétés de ces électrons peuvent être déterminées par la technique de photoémission résolue en angle [8]. Au cours d'une mesure de photoémission résolue en angle, un photon envoyé sur l'échantillon arrache un électron. On mesure alors la fonction spectrale du photo-trou ainsi créé. Pour un état électronique $|k\rangle$, la fonction spectrale $A(k, \omega)$ est reliée à la fonction de Green des électrons $G(k, \omega)$ :

$$
A(k, \omega)=-\frac{1}{\pi} \operatorname{Im} G(k, \omega) .
$$

Pour un système sans interaction, la fonction de Green $G_{o}(k, \omega)$ s'écrit :

$$
G_{o}(k, \omega)=\frac{1}{\omega-\xi_{k}+i \epsilon}
$$

$\xi_{k}$ représente la relation de dispersion des électrons (des fermions) prise par rapport au potentiel chimique $\mu$. Dans la limite où $\epsilon \rightarrow 0, A(k, w)=\delta\left(\omega-\xi_{k}\right)$.

Lorsque l'on augmente progressivement les interactions, toute particule dans un état de Bloch $|k, \sigma\rangle$ a une certaine probabilité d'être diffusée par collision avec une autre particule, laissant le système dans un état excité où des paires d'excitations électron-trou ont été créées. Dans un liquide de Fermi, les électrons sont remplacés par des quasiparticules caractérisées par une renormalisation de la relation de dispersion, de la masse effective et du temps de vie en comparaison des électrons sans interaction. Dans le cas d'interaction des fermions avec les fluctuations magnétiques (ou plus généralement des bosons) $[5,7]$, la fonction de Green en présence des interactions s'écrit : $\left.G(k, \omega)^{-1}\right)=G_{o}(k, \omega)^{-1}-\Sigma(k, \omega)$. 


\section{Collection SFN}

Le terme d'énergie propre $\Sigma(k, \omega)$ décrit les processus de diffusion des fermions avec les fluctuations magnétiques. La fonction spectrale mesurée par photo-émission prend une forme dans laquelle la renormalisation de la relation de dispersion des quasiparticules et leur amortissement sont directement reliés à $\Sigma(k, \omega)$ :

$$
A(k, \omega)=\frac{1}{\pi} \frac{\operatorname{Im} \Sigma(k, \omega)}{\left(\omega-\xi_{k}-\operatorname{Re} \Sigma(k, \omega)\right)^{2}+(\operatorname{Im} \Sigma(k, \omega))^{2}} ;
$$

et l'énergie propre $\Sigma(q, \omega)$ est déterminée par :

$$
\begin{aligned}
& \Sigma(k, \omega)=\frac{1}{\pi^{2}} \frac{1}{N} \sum_{q} \int_{-\infty}^{\infty} d \Omega d \nu V_{q k}^{2} \operatorname{Im} \chi(q, \Omega) \operatorname{Im} G(k+q, \nu)\left\{\frac{n^{B}(\Omega)+n^{F}(\nu)}{\omega+\Omega-v+i \epsilon}\right\} \\
& \Sigma(k, \omega)=-\frac{1}{\pi} \frac{1}{N} \sum_{q} \int_{-\infty}^{\infty} d \Omega V_{q k}^{2} \operatorname{Im} \chi(q, \Omega)\left\{\frac{n^{B}(\Omega)+n^{F}\left(\xi_{k+q}\right)}{\omega+\Omega-\xi_{k+q}+i \epsilon}\right\}
\end{aligned}
$$

$n^{B}(\Omega)=\left[\exp \left(\left(\Omega / k_{B} T\right)-1\right)\right]^{-1}$ est la distribution de Bose-Einstein. $\operatorname{Im} \chi(q, \omega)$ correspond à la partie imaginaire de la susceptibilité RPA. $V_{q k}$ est le terme de couplage spin-fermion qui est proportionnel $\sim U^{2}$. L'énergie propre contient tous les processus au cours desquels un électron est diffusé d'un état à un autre, moyennant l'émission ou l'absorption de fluctuations magnétiques.

\subsection{Spectre d'excitations des bosons}

Nous considérons à présent le cas d'un système présentant des excitations magnétiques localisées qui sont en interaction avec le bain d'électrons itinérants [5]. Il s'agit, par exemple, d'excitations de champ cristallin, mesurables par diffusion inélastique des neutrons. Un tel système est décrit à partir de l'Hamiltonien de basse énergie suivant :

$$
H=\sum_{k, \sigma} \xi_{k} c_{k \sigma}^{+} c_{k \sigma}+\sum_{q} \Omega_{q}\left(b_{q}^{+} b_{q}+\frac{1}{2}\right)-\frac{g}{N} \sum_{k, q} c_{k \alpha}^{+} \sigma_{\alpha \beta}^{z} c_{k+q \beta}\left(b_{-q}^{+}+b_{q}\right) .
$$

Les excitations magnétiques localisées sont caractérisées par une relation de dispersion $\Omega_{q}$ et les opérateurs bosoniques $b_{q}^{+}$et $b_{q}$ créent ou annihilent ces excitations. $g$ est le terme de couplage entre les excitations magnétiques localisées et les excitations de spin itinérantes.

Il est souvent pratique de définir un autre opérateur bosonique $\beta_{q}=\left(b_{-q}^{+}+b_{q}\right)$. Cet opérateur signifie que supprimer une excitation ayant pour vecteur de propagation $\mathbf{q}$ et une énergie $\Omega_{q}$ est equivalent à ajouter une excitation ayant pour vecteur de propagation $-\mathbf{q}$ et une énergie $-\Omega_{q}$ (cette excitation peut être vue comme l'anti-particule de la première).

La fonction de Green associé à cet opérateur de Boson est, en l'absence d'interaction :

$$
D^{o}(q, \omega)=\frac{1}{\omega-\Omega_{q}+i \epsilon}-\frac{1}{\omega+\Omega_{q}+i \epsilon}=\frac{2 \Omega_{q}}{\left(\omega^{2}-\Omega_{q}^{2}\right)+i \epsilon} .
$$

La fonction spectrale $-\frac{1}{\pi} \operatorname{Im} D^{o}(q, \omega)=\left[\delta\left(\omega-\Omega_{q}\right)-\delta\left(\omega+\Omega_{q}\right)\right]$ n'est autre que la partie imaginaire de la susceptibilité de spin localisé sans interaction. $D^{o}(q, \omega)$ représente essentiellement $-\chi_{L}(q, \omega)$ la susceptibilité localisée.

Sous l'effet du couplage avec les porteurs de charges, la fonction de Green bosonique est renormalisée et acquière une énergie propre, tel que $-D(q, \omega)^{-1}=-D^{o}(q, \omega)^{-1}-\Pi(q$, $\omega)$, où $\Pi$ est la fonction de polarisation.

$$
D(q, \omega)=\frac{2 \Omega_{q}}{\left.\left(\omega^{2}-\Omega_{q}^{2}\right)+2 \Omega_{q} \Pi(q, \omega)\right)}
$$


$\Pi(q, \omega)=g^{2} \chi^{o}(q, \omega)$ est relié à la susceptibilité sans interaction. Il est intéressant de noter que le couplage entre les fermions et les bosons renormalise le spectre des excitations bosoniques ainsi que le spectre des excitations fermioniques. On peut en effet calculer l'énergie propre des fermions à partir de l'équation Eq (2.12).

\section{ETAT SUPRACONDUCTEUR}

\subsection{Paires d'électrons et instabilité de la surface de Fermi [3]}

Dans un métal à température nulle, les électrons occupent les états en dessous du niveau de Fermi et les trous les états au dessus du niveau de Fermi. Au voisinage du niveau de Fermi, leur énergie $\xi_{k}$ peut être linéarisée : $\left|\xi_{k}\right|=v_{F}\left(k-k_{F}\right)$. On considère la possibilité de former des paires d'électrons au dessus du niveau de Fermi $\left(k>k_{F}\right)$ ou des paires de trous en dessous du niveau de Fermi $\left(k>k_{F}\right)$ sous l'effet d'une interaction attractive V. L'état fondamental n'ayant pas de courant, le centre de masse de la paire ne doit pas avoir d'impulsion, de telle sorte que les électrons doivent être caractérisés par des vecteurs d'onde de signes opposés. On suppose par ailleurs l'interaction attractive isotrope. L'état fondamental est spatialement symétrique et la fonction d'onde de spin antisymétrique $(l=0, \mathrm{~S}=0)$. L'équation de Schrödinger prend la forme :

$$
2\left|\xi_{k}\right| b_{k}+\sum_{k, k^{\prime}} V_{k, k^{\prime}} b_{k}^{\prime}=2 \Delta b_{k}
$$

Les termes $b_{k}$ représentent les amplitudes formant la paire de particules, $2 \Delta$ est l'énergie de liaison de la paire. $V_{k, k^{\prime}}$ est égal à $-V$, pour tous les états $k$ et $k^{\prime}$ pris dans une bande d'énergie de $\pm \omega_{D}$ autour du niveau de Fermi et $V_{k, k^{\prime}}$ est nulle dans les autres cas. On restreint le problème aux états k et k', tel que $k_{F}-\omega_{D} / v_{F}<|k|<k_{F}-\omega_{D} / v_{F}$ et on obtient alors :

$$
b_{k}=\frac{V}{2} \frac{A}{\left|\xi_{k^{\prime}}\right|+\Delta} \quad \text { avec } \quad A=\sum_{k^{\prime}} b_{k}^{\prime} .
$$

On peut ensuite déterminer $\Delta$ en sommant les amplitudes $b_{k}$. Aux vecteurs d'ondes au voisinage du niveau de Fermi, le spectre des particules peut être linéarisé et on ramène un problème tridimensionnel à un problème bidimensionnel. Cela a pour effet d'introduire une relation logarithmique entre $\Delta$ et $V$.

$$
1=\frac{V}{2} \sum_{k} \frac{1}{\left|\xi_{k^{\prime}}\right|+\Delta} \simeq \frac{V N_{F}}{2} \ln \left(\frac{\omega_{D}}{\Delta}\right)
$$

où $N_{F}$ représente la densité d'états au niveau de Fermi. L'énergie de liaison des paires s'écrit alors :

$$
2 \delta=2 \omega_{D} \exp \left(\frac{-2}{V N_{F}}\right) .
$$

On voit ici qu'on ne peut pas obtenir $\Delta$ par une méthode perturbative en V. Pour une interaction attractive $V$, aussi petite soit-elle, le niveau de Fermi devient instable par rapport à la formation de paires d'électrons.

\subsection{Supraconductivité : théorie BCS}

Un supraconducteur se caractérise par deux propriétés fondamentales : (i) il transporte un courant électrique sans aucune dissipation (résistivité électrique nulle), (ii) il est un système diamagnétique parfait (effet Meissner). En présence d'un champ magnétique extérieur, le système génère spontanément des super-courants qui écrantent le champ magnétique extérieur. 


\section{Collection SFN}

La théorie microscopique utilisée pour comprendre la supraconductivité est basée sur la théorie développée par Bardeen, Copper et Schrieffer (BCS) [3, 5]. La supraconductivité résulte de la formation de paires d'électrons (les paires de Cooper) en présence d'une interaction attractive entre électrons présents dans le système. Les paires de Cooper se forment à partir des états électroniques voisins du niveau de Fermi. Leur formation est un phénomène collectif, traduisant une instabilité globale de la surface de Fermi. Les paires de Cooper se forment toutes ensembles et se condensent simultanément dans un unique état quantique. Si une paire de Cooper peut être vue comme un boson composite formé de deux fermions, la supraconductivité est différente d'une condensation de Bose. En effet, dans la condensation de Bose, on considère un nombre de bosons fixé, dont une partie se condense dans un même état en dessous d'une température $T_{B}$. Pour un supraconducteur, le condensat de paires de Cooper se forme au même moment où les paires apparaissent à la température critique $T_{c}$. En dehors de l'état supraconducteur les paires de Cooper ne sont pas stables.

L'état fondamental supraconducteur se caractérise par la fonction d'onde :

$$
\left|\Psi_{B C S}>=\prod\left(u_{k}+v_{k}\right) c_{k, \uparrow}^{+} c_{-k, \downarrow}^{+}\right| 0>
$$

avec :

$$
\left|u_{k}\right|^{2}=\frac{1}{2}\left(1+\frac{\xi_{k}}{\left|E_{k}\right|}\right) \quad\left|v_{k}\right|^{2}=\frac{1}{2}\left(1-\frac{\xi_{k}}{\left|E_{k}\right|}\right) \quad u_{k}^{\star} v_{k}=\frac{1}{2} \frac{\Delta_{k}}{\left|E_{k}\right|}
$$

et $\Delta_{k}$ est donné par l'équation du «gap» :

$$
\Delta_{k}=-\frac{1}{N} \sum_{q} V_{q} \frac{\Delta_{k-q}}{2 E_{k-q}}
$$

où $V_{q}$ représente le potentiel d'interaction entre électrons. $E_{k}= \pm \sqrt{\xi_{k}^{2}+\left|\Delta_{k}\right|^{2}}$ correspond à l'énergie des excitations élémentaires $\gamma_{ \pm k}(E<0)$ et $\gamma_{ \pm k}^{+}(E<0)$ qui apparaissent à partir de l'état fondamental. Ces excitations se présentent sous la forme d'un combinaison d'un électron et d'un trou et sont donc des fermions.

$$
\begin{aligned}
& \gamma_{k}=u_{k}^{\star} c_{k \uparrow}-v_{k}^{\star} c_{-k \downarrow}^{+} \\
& \gamma_{-k}=u_{k}^{\star} c_{-k \downarrow}+v_{k}^{\star} c_{k \uparrow}^{+} \\
& \gamma_{k}^{+}=u_{k} c_{k \uparrow}^{+}-u_{k} c_{-k \downarrow} \\
& \gamma_{-k}^{+}=u_{k} c_{-k \downarrow}^{+}+v_{k} c_{k \uparrow} .
\end{aligned}
$$

Notons qu'en général l'ouverture d'un gap dans le spectre d'excitations électroniques entraine un état isolant. Dans un supraconducteur, ce ne sont pas les états électroniques à une particule qui sont importants. Ils peuvent être vus comme des états excités correspondant à des paires brisées. Pour comprendre les propriétés de transport d'un supraconducteur, il faut garder à l'esprit que le système se caractérise par une fonction d'onde macroscopique ayant une phase unique. Lorsqu'un courant passe [5], la phase va légèrement varier sur une distance macroscopique. Le courant qui circule est alors proportionnel au gradient de la phase de la fonction d'onde supraconductrice. Dans ce cas, ce sont les électrons du condensat de paires de Cooper qui vont transporter le courant. Pour que le système devienne résistif, il faudrait que les impuretés présentent dans le système soient capables de diffuser l'ensemble des électrons du condensat, c'est à dire un nombre d'électrons macroscopique. Dans un état supraconducteur, le fait de fixer la phase des électrons (cohérence de phase) est équivalent à fixer la direction des spins dans un état ferromagnétique. 


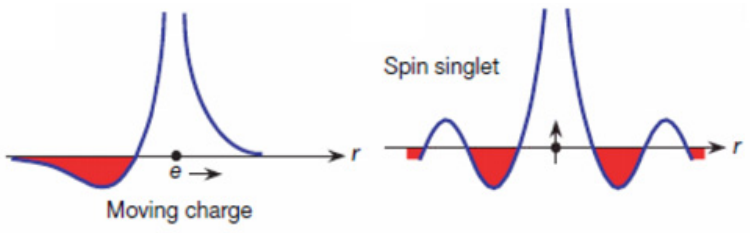

Border of antiferromagnetism

a)

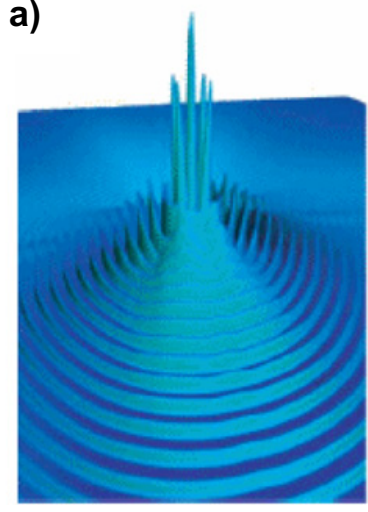

b)

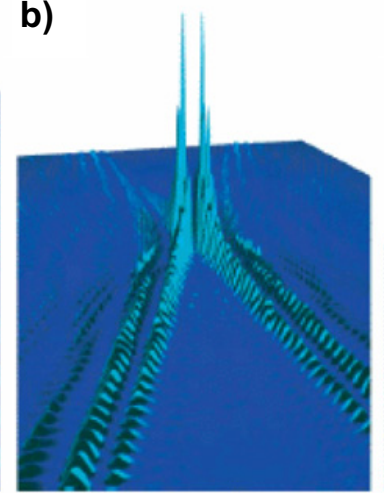

Spin triplet

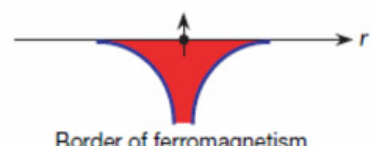

c)

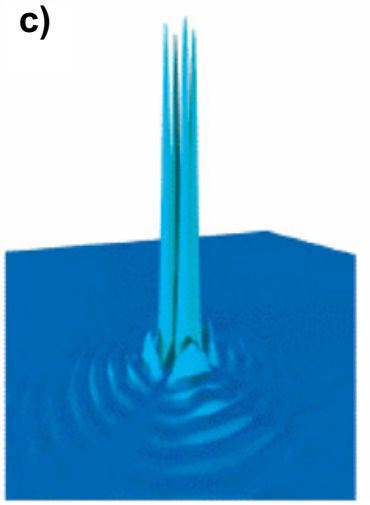

Figure 2. La figure montre la nature du potentiel entre électrons dans l'espace réel. La couleur rouge indique que le potentiel est attractif. La figure montre également la probabilité de trouver le second électron d'une paire de Cooper à une distance $r$ du premier électron situé à l'origine. Cette fonction présente de petites oscillations qui correspondent à l'ensemble des états électroniques autour du niveau de Fermi qui contribuent à la fonction de paire. a) Couplage par échange de phonons - supracondtivité singulet de symétrie $s$, b) couplage par échange de fluctuations antiferromagnétique - supracondtivité singulet de symétrie $d$, c) couplage par échange de fluctuations ferromagnétiques - supracondtivité triplet de symétrie $p$. [6].

\subsection{Supraconductivité : origine du potentiel attractif :}

La théorie BCS est une théorie microscopique qui permet de comprendre l'origine de la supraconductivité dans les supraconducteurs dits «conventionnels». Dans ces matériaux, la température critique supraconductrice $T_{c}$ varie avec la racine carrée de la masse des atomes. C'est ce que l'on appelle l'effet isotopique. Cela indique que les phonons ont un rôle crucial dans l'apparition de la supraconductivité. Cooper a ainsi montré qu'une interaction attractive apparaissait entre les électrons par un mécanisme d'échange de phonons : un premier électron émet un phonon qui est ensuite absorbé par un second électron. En effet, un électron de charge négative peut induire un déplacement des ions positifs du réseau cristallin. Une fois l'électron parti, les ions plus massifs ont une certaine inertie et ne reviennent pas immédiatement à leur position d'équilibre. Il subsiste donc une concentration de charges positives qui peut attirer un second électron dans le sillage du premier. Dans cette image, l'interaction n'est pas instantané (Fig. 2a).

Ce mécanisme engendre un potentiel attractif et isotrope jusqu'à une énergie caractéristique qui est $\omega_{D}$ la fréquence de Debye des phonons. La théorie BCS complète la description de l'état supraconducteur en proposant une fonction d'onde variationnelle $\mid \Psi_{B C S}>$ qui décrit l'état fondamental supraconducteur. A partir de cet état fondamental, toutes les propriétés thermodynamiques et les propriétés de transport ont pu être calculées, donnant un accord remarquable avec les observations expérimentales. Dans la théorie BCS, le potentiel d'interaction $V_{q}=-g$ est attractif et isotrope. On peut donc trouver un solution à l'équation du gap Eq. (3.25), tel que $\Delta_{k}$ est indépendant de $k$. C'est 


\section{Collection SFN}

la solution qui correspond à une fonction de paire de symétrie s $(l=0)$ et singulet de spin $(S=0)$ (Fig. 2a).

On peut étendre le modèle proposé par BCS, mais en s'intéressant cette fois à une supraconductivité sans phonon [6]. Les électrons pourraient ainsi s'apparier par l'échange de fluctuations qui ne sont pas liés au réseau cristallin. Par exemple, nous avons vu au chapitre précédent que sous l'effet des interactions électroniques, les électrons engendraient des fluctuations magnétiques ou pouvaient être couplés à un sous-système de fluctuations magnétiques localisées. Nous pouvons donc envisager deux mécanismes d'appariement supraconducteur. Dans un premier cas, nous avons les électrons et un soussystème magnétique localisé dont les excitations vont se substituer aux phonons. Dans un second cas, on considère un système d'électrons en interaction. Ceux-ci engendrent des fluctuations magnétiques qu'ils peuvent ensuite échanger. Dans ce dernier cas, la supraconductivité est d'origine purement électronique. Dans tous les cas, nous conservons l'idée qu'un électron peut polariser le milieu dans lequel il évolue et que cette polarisation se comporte comme un champ attractif pour un second électron.

En présence d'une répulsion coulombienne U, un potentiel $V_{q} \simeq \frac{3}{2} U^{2} \chi^{R P A}(q, \omega)$ intervient dans la formation de paires de Cooper singulet. Dans ce cas, l'équation du gap Eq. (3.25) n'a de solution que si l'on autorise $\Delta_{k}$ à changer de signe. Si $Q$ est le vecteur caractéristique des fluctuations magnétiques, alors on cherchera un solution tel que $\Delta_{k+Q}=-\Delta_{k}$. On peut a priori obtenir une supraconductivité singulet de spin, mais non isotrope, par exemple de symétrie d $(l=2)$. Ce cas est en général considéré lorsque le système est proche d'instabilité antiferromagnétique. (Fig. 2b). Aux fluctuations antiferromagnétiques est associé un potentiel attractif tantôt attractif, tantôt répulsif, tout va dépendre des sites sur lesquels se trouvent les électrons (Fig. 2b).

De même, on peut considérer le cas d'un état supraconducteur qui n'est plus singulet de spin $(\mathrm{S}=0)$ mais triplet $(S=1)$. La fonction d'onde supraconductrice n'est alors pas isotrope. Elle peut par exemple être de symétrie $\mathrm{p}(l=1)$. On s'intéresse en général à la présence de fortes fluctuations ferromagnétiques (Fig. 2c) et un potentiel d'interaction attractif de la forme $V_{q} \simeq-\frac{1}{2} U^{2} \chi^{R P A}(q, \omega)$.

En présence d'un état supraconducteur non conventionnel (paramètre d'ordre supraconducteur singulet mais anisotrope, ou triplet), l'étude de spectre des excitations magnétiques dans l'état normal par diffusion inélastique de neutrons peut donc fournir des indices permettant de rechercher un mécanisme d'appariement supraconducteur non conventionnel faisant intervenir les fluctuations magnétiques du système. Cependant, le problème est moins simple qu'il n'y parait. Pour deux raisons : (i) le spectre des excitations magnétiques est modifié en passant de l'état normal à l'état supraconducteur, (ii) le potentiel d'interaction est relié à la susceptibilité magnétique qui est très dépendante de $\mathbf{q}$ et $\omega$.

\subsection{Susceptibilité de spin dans un supraconducteur singulet}

\section{- Susceptibilité sans interaction :}

La susceptibilité de spin est décrite à partir d'excitations électron-trou. Dans l'état supraconducteur, il en est de même, mais il faut à présent exprimer les opérateurs $c_{k, \sigma}$ et $c_{k, \sigma}^{+}$en fonction des opérateurs de quasiparticules $\gamma_{ \pm k}$ et $\gamma_{ \pm k}^{+}$caractéristiques de l'état suspraconducteur. La susceptibilité de spin sans interaction, correspondant à la susceptibilité Lindhard dans l'état normal, est à présent donnée par la susceptibilité BCS :

$$
\chi^{o}(q, \omega)=-\frac{1}{N} \sum_{k} \sum_{\alpha, \beta= \pm} C_{q k}^{\alpha \beta} \frac{n^{F}\left(\alpha E_{k}\right)-n^{F}\left(\beta E_{k+q}\right)}{\omega+\alpha E_{k}-\beta E_{k}+i \epsilon}
$$


où apparait le facteur de cohérence :

$$
C_{q k}^{\alpha \beta}=\frac{1}{4}\left(1+\alpha \beta \frac{\xi_{k+q} \xi_{k}+\Delta_{k+q}^{\star} \Delta_{k}}{E_{k+q} E_{k}}\right) .
$$

Afin de voir de quelle façon, la supraconductivité modifie la susceptibilité sans interaction, nous nous plaçons à température nulle et considérons la partie imaginaire de la susceptibilité magnétique pour $\omega>0$

$$
\operatorname{Im} \chi^{o}(q, \omega>0) \sim \sum_{k} \frac{1}{4}\left(1-\frac{\xi_{k+q} \xi_{k}+\Delta_{k+q}^{\star} \Delta_{k}}{E_{k+q} E_{k}}\right) \delta\left(\omega-E_{k+q}-E_{k}\right) .
$$

Nous voyons que le continuum d'excitations de Stoner apparait au dessus d'une énergie seuil $\omega_{c}=$ $\min \left[E_{k+q}+E_{k}\right]\left(\sim\left(\left|\Delta_{k}\right|+\left|\Delta_{k+q}\right|\right)\right)$. Pour engendrer des excitations magnétiques, il faut donc casser des paires de Cooper, ce qui implique de fournir un minimum d'énergie au système. Si l'on considère à présent, $\xi_{k+q}=\xi_{k}=0$, nous voyons que le facteur de cohérence est nul lorsque est $\Delta_{k+q}^{\star} \Delta_{k}<0$ et égal

à $\frac{1}{2}$ dans le cas contraire. Le poids spectral des excitations reflète le changement de signe du paramètre d'ordre supraconducteur avec $\mathrm{k}$. Mesurer la susceptibilité magnétique revient à réaliser une mesure d'interférence qui sonde les changements de signe de $\Delta_{k}$.

\section{- Susceptibilité RPA :}

Si l'on prend en compte les interactions répulsives entre électrons (U), la susceptibilité en interaction dans l'état supraconducteur est donnée comme dans l'état normal par la susceptibilité RPA (Eq. (2.5)), mais c'est la forme de la susceptibilité sans interaction qui est à présent différente. La partie imaginaire de la susceptibilité RPA s'écrit :

$$
\operatorname{Im} \chi^{R P A}(q, \omega)=\frac{\operatorname{Im} \chi^{o}(q, \omega)}{\left(1-U \operatorname{Re} \chi^{o}(q, \omega)\right)^{2}+\left(U \operatorname{Im} \chi^{o}(q, \omega)\right)^{2}} .
$$

En dessous de la bordure inférieure du continuum de Stoner $\left(\omega_{c}\right), \operatorname{Im} \chi^{o}(q, \omega)$ est toujours nulle. Si pour une énergie $\Omega_{q}<\omega_{c},\left(1-U \operatorname{Re} \chi^{o}\left(q, \Omega_{q}\right)\right)$ s'annule, alors :

$$
\operatorname{Im} \chi^{R P A}(q, \omega>0)=-\pi\left[U \frac{d \operatorname{Re} \chi^{o}(q, \omega)}{d \omega}\right]_{\omega \rightarrow \Omega_{q}}^{-1} \delta\left(\omega-\Omega_{q}\right) .
$$

Cette excitation se trouvant en dessous du continuum de Stoner, elle ne correspond pas à une excitation élémentaire. Il s'agit d'une excitation magnétique collective, poussée en dessous du continuum de Stoner sous l'effet des corrélations électroniques (Fig. 3). Cette excitation traduit la formation d'un état lié electron-trou, appelé exciton de spin. Cette excitation ne transporte pas de charge, se propage avec un vecteur d'onde q et transporte une quantité de spin $S=1$. Elle n'existe qu'en dessous du continuum de Stoner et se redécompose en excitation électron-trou élementaires en entrant dans le continuum de Stoner. Au dessus de la température critique supraconductrice $T_{c}$, l'exciton de spin disparait. Cette excitation magnétique est l'analogue des magnons que l'on trouve aussi en dessous du continuum de Stoner dans les systèmes ferromagnétiques itinérants ou les systèmes à onde de densité de spin.

\subsection{Susceptibilité de spin dans un supraconducteur triplet}

\section{- Supraconductivité triplet de symétrie $p$}

Avant de discuter l'évolution du spectre des excitations magnétiques dans l'état supraconducteur, il convient de donner quelques notions utiles permettant de mieux appréhender la supraconductivité triplet [12]. La description de la supraconductivité triplet de symétrie $p$ a été mené par analogie avec la 


\section{Collection SFN}
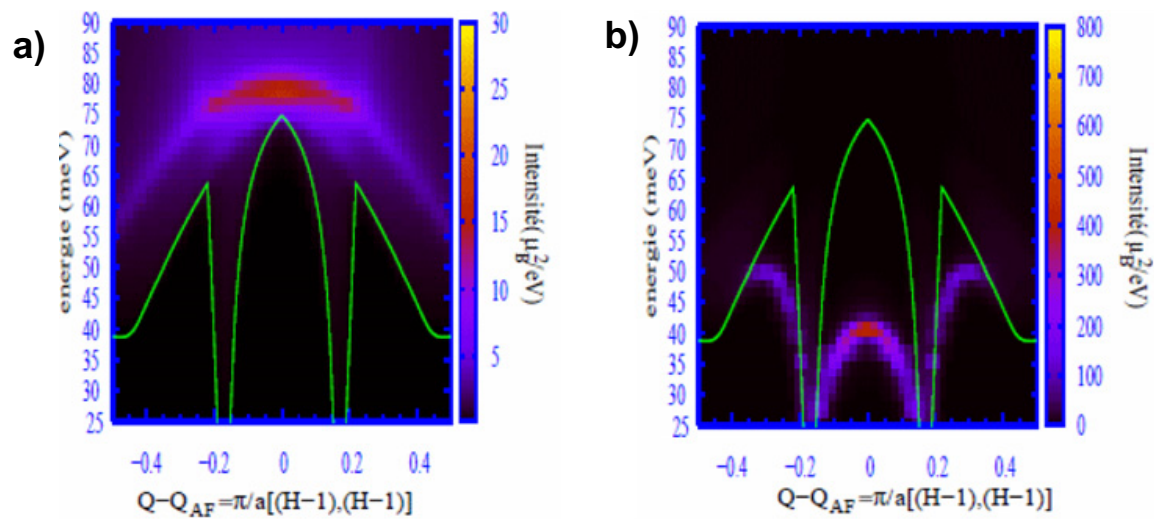

Figure 3. Partie imaginaire de la susceptibilité de spin autour du vecteur d'onde antiferromagnétique $\mathbf{Q}_{A F}$. La susceptibilité sans interaction dans l'état supraconducteur est calculée en prenant une relation de dispersion $\xi_{k}=$ $-2 t\left(\cos \left(k_{x}\right)+\cos \left(k_{y}\right)\right)+4 t^{\prime} \cos \left(k_{x}\right) \cos \left(k_{y}\right)-\mu$ et un gap supraconducteur de symétrie $d$, tel $\Delta_{k}=\frac{\Delta}{2}\left(\cos \left(k_{x}\right)-\right.$ $\left.\cos \left(k_{y}\right)\right)$. La ligne verte représente $\omega_{c}$ la bordure inférieure du continuum de Stoner. a) Susceptibilité sans interaction $(U=0)$, b) susceptibilité RPA $(U \neq 0)[9]$.

description théorique de la superfluidité dans $1{ }^{3} \mathrm{He}$. Pour $1{ }^{3} \mathrm{He}$, il n’y pas de réseau et les espaces des spins et des orbites sont découplés. Les paires de Cooper sont alors caractérisés par le moment de spin $(S)$ et leur moment angulaire orbital $(l)$. Les paires de Cooper doivent posséder un moment total pair : $S=1$ et $l=1,3$, etc....

De façon générale, le paramètre d'ordre supraconducteur $\Delta(\mathbf{k})$ peut être décrit sous une forme matricielle :

$$
\Delta(\mathbf{k})=\left(\begin{array}{cc}
\Delta_{\uparrow \uparrow} & \Delta_{\uparrow \downarrow} \\
\Delta_{\downarrow \uparrow} & \Delta_{\downarrow \downarrow}
\end{array}\right)
$$

pour un supraconducteur singulet, $\Delta_{\uparrow \uparrow}=\Delta_{\downarrow \downarrow}=0$ et $\Delta_{\uparrow \downarrow}=-\Delta_{\downarrow \uparrow}=\Delta_{s}$, et pour un supraconducteur triplet, $\Delta_{\uparrow \downarrow}=\Delta_{\downarrow \uparrow}=\Delta_{0}$. Un des concepts fort utile pour décrire la supraconductivité triplet est celui du vecteur $\mathbf{d}(\mathbf{k})=\left[d_{x}(\mathbf{k}), d_{y}(\mathbf{k}), d_{z}(\mathbf{k})\right]$ :

$$
\Delta(\mathbf{k})=\left(\begin{array}{ll}
\Delta_{\uparrow \uparrow} & \Delta_{\uparrow \downarrow} \\
\Delta_{\downarrow \uparrow} & \Delta_{\downarrow \downarrow}
\end{array}\right)=\left(\begin{array}{lr}
-d_{x}+i d_{y} & d_{z} \\
d_{z} & d_{x}+i d_{y}
\end{array}\right)=i\left(\sum_{\nu} d^{\nu} \sigma_{\nu}\right) \sigma_{y}
$$

$\sigma_{v}$ correspondent aux matrices de Pauli $(v=x, y, z)$. Le vecteur $\mathbf{d}(\mathbf{k})$ permet de décrire les symétries de l'état supraconducteur, le moment de spin et le moment orbital des paires de Cooper, ainsi que les noeuds dans le gap supraconducteur. La fonction d'onde d'une paire de Cooper $|\psi\rangle$ s'écrit :

$$
\left|\psi>=\Delta_{\uparrow \uparrow}\right| \uparrow \uparrow>+\Delta_{\downarrow \downarrow} \mid \downarrow \downarrow>+\Delta_{0}(|\uparrow \downarrow>+| \downarrow \uparrow>)
$$

où la base $|\uparrow \uparrow>,| \downarrow \downarrow>$ et $\frac{1}{\sqrt{2}}(|\uparrow \downarrow>+| \downarrow \uparrow>)$ correspond aux projections des spins des paires de Cooper $S_{z}=1,-1$ et 0 . Pour donner toute sa signification au vecteur $\mathbf{d}$, on peut introduire une nouvelle base :

$$
\begin{aligned}
& \mathbf{z}=\mid S_{z}=0>=\frac{1}{\sqrt{2}}(|\uparrow \downarrow>+| \downarrow \uparrow>) \\
& \mathbf{x}=\mid S_{x}=0>=\frac{1}{\sqrt{2}}(-|\uparrow \uparrow>+| \downarrow \downarrow>) \\
& \mathbf{y}=\mid S_{y}=0>=\frac{1}{\sqrt{2}}(|\uparrow \uparrow>+| \downarrow \downarrow>)
\end{aligned}
$$


a)

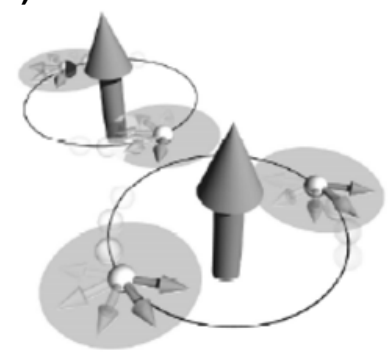

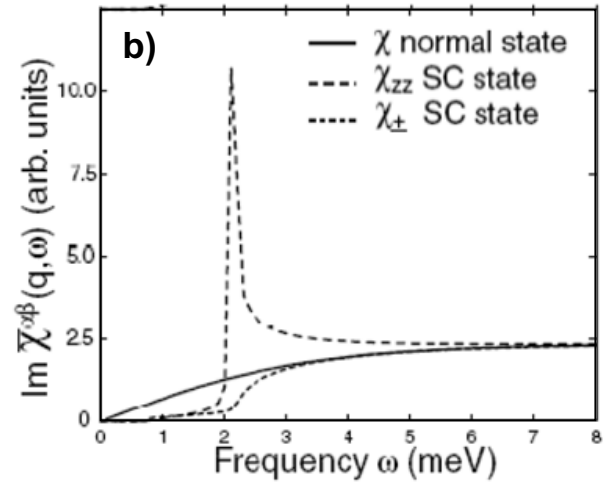

Figure 4. a) Description schématique des vecteurs $\mathrm{S}$ et $\mathrm{L}$ des paires de Cooper pour un paramètre d'ordre de symétrie $p$ avec $\mathbf{d}=\mathbf{z}\left(\sin \left(k_{x}\right) \pm i \sin \left(k_{y}\right)\right)$. Les spins sont alignés perpendiculairement au vecteur $\mathbf{d}\left(\mid S=1 ; S_{z}=\right.$ $0\rangle)$. Le moment orbital est parallèle au vecteur $\mathbf{d}$ et est deux fois dégénéré $\left(\left|L=1 ; L_{z}= \pm 1\right\rangle\right.$ [12]. Cet état supraconducteur est l'analogue de la phase ABM pour $1{ }^{3} \mathrm{He}$ superfluide. b) Partie imaginaire de la susceptibilité RPA.

de telle sorte que la fonction d'onde $\mid \psi>$ puisse s'écrire en fonction des composantes du vecteur d dans cette nouvelle base :

$$
\mid \psi>=\sqrt{2}\left(d_{x} \mathbf{x}+d_{y} \mathbf{y}+d_{z} \mathbf{z}\right)
$$

Le vecteur $\mathbf{d}$ indique donc la direction suivant laquelle la projection du spin des paires de Cooper est nulle (Fig. 3a).

L'énergie des quasiparticules excitées est donnée par :

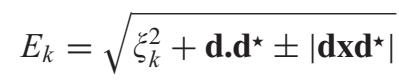

$\xi_{k}$ est l'énergie des quasiparticules dans l'état normal, prise par rapport au niveau de Fermi. Lorsque $\left|\mathbf{d x d}^{\star}\right|=0$, l'état est dit unitaire et on montre facilement que $\mathbf{d . d} \mathbf{d}^{\star}$ n'est autre que $|\Delta(\mathbf{k})|^{2}$. Notons que dans le cas non-unitaire, le spectre des quasiparticules excités présente deux gaps.

\section{- Spectre des excitations magnétiques dans l'état supraconducteur}

Joynt et Rice [10] furent les premiers à souligner que les dépendences en énergie et en vecteur d'onde de la susceptibilité magnétique reflètaient directement la dépendance en vecteur d'onde du gap supraconducteur et que sa mesure par diffusion inélastique de neutrons avait le potentiel de permettre une identification complète de la symétrie du gap supraconducteur.

Dans l'état supraconducteur la susceptibilité magnétique sans interaction n'est plus simplement décrite par la fonction de Lindhard. A $\mathrm{T} \rightarrow 0$ et pour des énergies positives, $\chi^{o}(\mathbf{q}, \omega)$ s'écrit [10] :

$$
\chi_{\alpha \beta}^{o}(\mathbf{q}, \omega)=-\frac{1}{N} \sum_{\mathbf{k}} \frac{C_{\alpha \beta}(\mathbf{k}, \mathbf{q})}{\omega+i \epsilon-E_{+}-E_{-}}
$$




\section{Collection SFN}

$E_{ \pm}=\sqrt{\left(\xi_{\mathbf{k} \pm \mathbf{q} / \mathbf{2}}\right)^{2}+\mid \Delta^{\star}\left(\mathbf{k} \pm \mathbf{q} /\left.\mathbf{2}\right|^{2}\right.}$ est l'énergie des quasiparticules excitées et $C_{\alpha \beta}(\mathbf{k}, \mathbf{q})$ correspond au facteur de cohérence :

$$
C_{\alpha \beta}(\mathbf{k}, \mathbf{q})=\frac{\left.\frac{1}{2} \delta_{\alpha \beta}\left[\left(E_{+}+\xi_{+}\right)\left(E_{-}-\xi_{-}\right)+\operatorname{Re}\left(\mathbf{d}_{+}^{\star} \mathbf{d}_{-}\right)\right)\right]-\operatorname{Re}\left(d_{\alpha+}^{\star} d_{\beta-}\right)}{2 E_{+} E_{-}} .
$$

Comme dans le cas singulet, nous voyons que le gap supraconducteur apparait explicitement dans la susceptibilité magnétique sans interaction. De plus, au travers du facteur de cohérence, la susceptibilité peut être soit amplifiée, soit réduite suivant la nature du paramètre d'ordre supraconducteur. Dans la mesure où le vecteur $\mathbf{d}$ est aligné le long d'une direction particulière, l'effet du facteur de cohérence va être de pouvoir rendre la susceptibilité magnétique anisotrope dans l'espace des spins et ceci même si la susceptibilité est parfaitement isotrope dans l'état normal. Pour $\mathbf{d}$ parallèle à $\mathbf{z}$, les facteurs de cohérence deviennent :

$$
\begin{aligned}
C_{x x, y y}(\mathbf{k}, \mathbf{q}) & =\frac{1}{4} \frac{\left(E_{+}+\xi_{+}\right)\left(E_{-}-\xi_{-}\right)+\left(\Delta_{+}^{\star} \Delta_{-}\right)}{E_{+} E_{-}} \\
C_{z z}(\mathbf{k}, \mathbf{q}) & =\frac{1}{4} \frac{\left(E_{+}+\xi_{+}\right)\left(E_{-}-\xi_{-}\right)-\left(\Delta_{+}^{\star} \Delta_{-}\right)}{E_{+} E_{-}} .
\end{aligned}
$$

Ces effets d'anisotropie croissent lorsque que les corrélations électroniques sont introduites dans une approche RPA (Fig. 3b).

\subsection{Spectre des quasiparticules et instabilité supraconductrice :}

Dans l'état supraconducteur, on considère deux types de fonctions de Green, dites normale et anormale : $G(k, \omega)$ et $F(k, \omega)$, respectivement. Ces fonctions décrivent les propriétés des quasiparticules et des paires de Cooper.

$$
\begin{gathered}
G(k, \omega)=\frac{u_{k}^{2}}{\omega-E_{k}+i \epsilon}+\frac{v_{k}^{2}}{\omega+E_{k}+i \epsilon}=\frac{\omega+\xi_{k}}{\omega^{2}-\xi_{k}^{2}-\Delta_{k}^{2}+i \epsilon} \\
F(k, \omega)=-u_{k} v_{k}\left(\frac{1}{\omega-E_{k}+i \epsilon}-\frac{1}{\omega+E_{k}+i \epsilon}\right)=\frac{\Delta_{k}}{\omega^{2}-\xi_{k}^{2}-\delta_{k}^{2}+i \epsilon} .
\end{gathered}
$$

La valeur de $\Delta_{k}$ est donné par l'équation du gap, qui à température finie est :

$$
\Delta_{k}=-\frac{1}{N} \sum_{q} V_{q} \frac{\Delta_{k-q}}{2 E_{k-q}} \operatorname{th}\left(\frac{E_{k-q}}{k_{B} T}\right) .
$$

Le fait que les électrons interagissent avec des fluctuations (de réseau, ou de spin par exemple) et que ces fluctuations ont une dépendance en énergie et vecteur d'onde spécifique disparait. Pour des fluctuations magnétiques, cela équivaut à prendre $V_{q} \sim \frac{3}{2} U \chi(q, 0)$. Cependant de façon générale, on doit prendre en compte la dépendance en énergie du couplage entre les électrons : $V(q, \omega) \sim \frac{3}{2} U \chi(q, \omega)$. $\xi_{k}$ et $\Delta_{k}$ se mettent alors à dépendre de l'énergie :

$$
\begin{aligned}
\xi_{k} & \rightarrow \bar{\xi}(k, \omega)=\xi_{k}+\Sigma(k, \omega) \\
\Delta_{k} & \rightarrow \Phi(k, \omega) .
\end{aligned}
$$



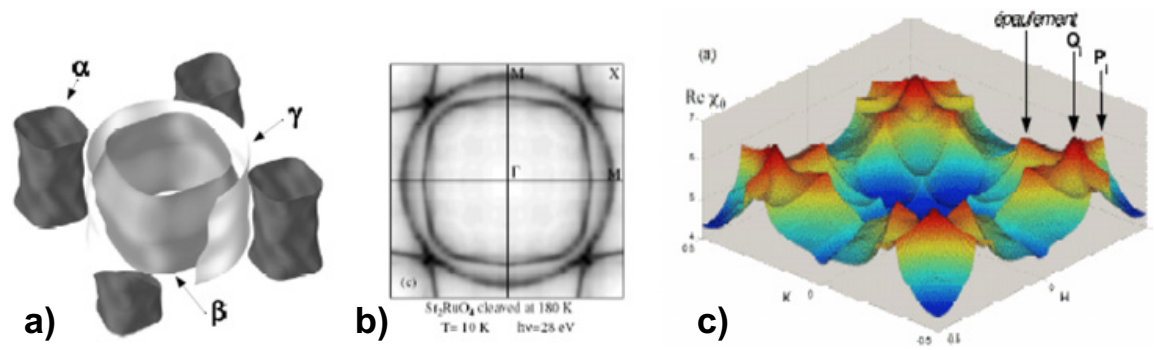

Figure 5. a) Surfaces de Fermi déterminées par mesures de de Haas van Alphen (Ref. [12], voir C. Bergemann al.). b) Mesure des surfaces de Fermi par photoémission résolue en angle [13]. c) Calcul de la susceptibilité sans interaction [14].

Pour trouver la dépendance en énergie de $\Sigma$ et $\Phi$ il faut résoudre un jeu d'équations non linéaires, les équations d'Eliasberg généralisées [11]:

$$
\begin{aligned}
G(k, \omega) & =\frac{\omega+\bar{\xi}(k,-\omega)}{(\omega-\bar{\xi}(k, \omega))(\omega+\bar{\xi}(k,-\omega))-|\Phi(k, \omega)|^{2}+i \epsilon} \\
F(k, \omega) & =\frac{\Phi(k, \omega)}{(\omega-\bar{\xi}(k, \omega))(\omega+\bar{\xi}(k,-\omega))-|\Phi(k, \omega)|^{2}+i \epsilon} \\
\Sigma(k, \omega) & =\frac{1}{\pi^{2}} \frac{1}{N} \sum_{q} \int_{-\infty}^{\infty} d \Omega d \nu V_{q k}^{2} \operatorname{Im} \chi(q, \Omega) \operatorname{Im} G(k+q, v) \frac{n_{B}(\Omega)+n_{F}(v)}{\omega+\Omega-v+i \epsilon} \\
\Phi(k, \omega) & =\frac{1}{\pi^{2}} \frac{1}{N} \sum_{q} \int_{-\infty}^{\infty} d \Omega d v V_{q k}^{2} \operatorname{Im} \chi(q, \Omega) \operatorname{Im} F(k+q, v) \frac{n_{B}(\Omega)+n_{F}(v)}{\omega+\Omega-v+i \epsilon} .
\end{aligned}
$$

Le problème est loin d'être simple à résoudre, mais la détermination de $\operatorname{Im} \chi(q, \Omega)$ expérimentalement par diffusion inélastique des neutrons prend alors une importance capitale.

\section{MESURE DE DIFFUSION INÉLASTIQUE DES NEUTRONS : ÉTAT NORMAL}

\subsection{Structure électronique du composé $\mathrm{Sr}_{2} \mathrm{RuO}_{4}$}

Le composé $\mathrm{Sr}_{2} \mathrm{RuO}_{4}$ [12] présente une structure de type perovskite en couche. Il se caractérise par l'empilement de plans $\mathrm{RuO}_{2}$. En dessous de $1.5 \mathrm{~K}$, ce matériau devient un supraconducteur triplet de symétrie $p$ [12]. Il est souvent présenté comme l'analogue électronique et quasi-2D de $1^{1}{ }^{3} \mathrm{He}$ superfluide. Tout comme $1^{1} \mathrm{He}$, l'appariement des fermions pourrait être induit par l'échange de fortes fluctuations ferromagnétiques. Le spectre des excitations magnétiques du composé $\mathrm{Sr}_{2} \mathrm{RuO}_{4}$ n'est cependant pas dominé par des fluctuations ferromagnétiques, mais par des fluctuations incommensurables dont l'origine est parfaitement comprise dans une approche de magnétisme itinérant.

La surface de Fermi et la structure de bande du composé $\mathrm{Sr}_{2} \mathrm{RuO}_{4}$ ont bien été étudiés expérimentalement [12,13] et théoriquement [15], avec un accord remarquable entre la théorie et l'expérience. Quatre électrons sont présents sur l'ion $R u^{4+}$ et répartis équitablement entre les trois orbitales $t_{2 g}$. Les orbitales $4 d_{x y}$ sont bien hybridées dans le plan $\mathrm{RuO}_{2}$ et donnent naissance à une bande qui présente un fort caractère bidimensionnel : la bande $\gamma$ (Fig. 5). A l'opposé, les 
a)

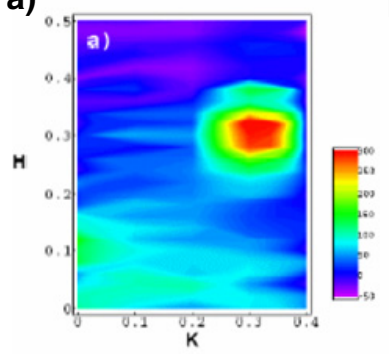

b)

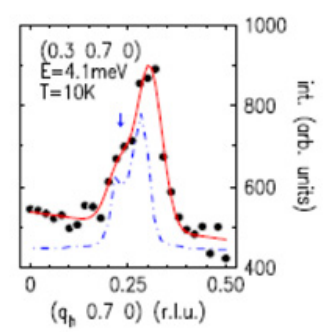

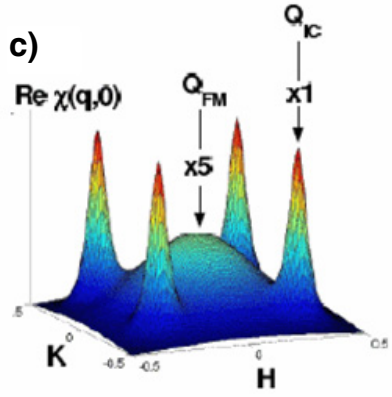

Figure 6. a) Cartographie de l'intensité magnétique pour un transfert d'énergie de $4.1 \mathrm{meV}$ [18]. b) Coupe à énergie constante le long de la direction $(1,0,0)$ au travers du pic incommensurable. c) Dépendance en fonction du moment de la partie réelle de la susceptibilité magnétique $(\omega=0)$, déduite des mesures de diffusion inélastique des neutrons [14].

orbitales $4 d_{x z}$ et $4 d_{y z}$ ne s'hybrident que le long des directions $x$ ou $y$ et donnent lieu à des bandes quasi-unidimensionnelles : les bandes $\alpha$ et $\beta$ (Fig. 5). Le caractère quasi-unidimensionnel des bandes $\alpha$ et $\beta$ leur permet de s'emboiter les unes dans les autres. Cet effet de nesting conduit à une forte augmentation de la susceptibilité sans interaction pour des vecteurs d'onde $\mathbf{q}=\left(0.33, q_{y}, 0\right)$ ou $\mathbf{q}=$ $\left(q_{x}, 0.33,0\right)$ [16]. Le long des diagonales, les deux effets se cumulent, donnant lieu à des maxima dans la susceptibilité sans interaction $\chi^{o}$ pour $\mathbf{q}_{\mathbf{i}}=( \pm 0.33, \pm 0.33,0)$. Le calcul suggère également l'existence de lignes de forte susceptibilité le long de $\left(0.33, q_{y}, 0\right)$ pour $0.33<q_{y}<0.5$, ainsi que des épaulements pour $0<q_{y}<0.33$ (Fig. 5c).

\subsection{Mesures de diffusion inélastique dans l'état normal du composé $\mathrm{Sr}_{2} \mathrm{RuO}_{4}$}

Les mesures de diffusion inélastique des neutrons non polarisés [17, 18] ont confirmé ce scénario en montrant un spectre d'excitations magnétiques dominé à basse énergie par des fluctuations magnétiques $2 \mathrm{D}$ au vecteur d'onde $\mathbf{q}_{I C}=(0.3,0.3, L)$ (Fig. 6a). Comme dans les calculs, un épaulement est également observé à côté du pic principal (Fig. 6b). Afin de comparer de façon plus précise, la théorie et l'expérience, la susceptibilité RPA a été calculée en prenant une interaction effective $U_{e f f}(\mathbf{q})=\frac{320 m e V}{1+0.08 \mathbf{q}^{2}}$ associée à la répulsion coulombienne sur les sites du ruthénium et de l'oxygène. Parallèlement la susceptibilité magnétique mesurée a été calibrée en unité absolue $\left(\mu_{B}^{2} \cdot \mathrm{eV}^{-1}\right)$. On en déduit le facteur d'augmentation de Stoner :

$$
\frac{1}{\delta}=\frac{\chi^{\text {neutron }}\left(q_{I C}\right)}{\chi^{o}\left(q_{I C}\right)} \frac{1}{1-U_{e f f}\left(q_{I C}\right) \chi^{o}\left(q_{i}\right)}=1+U_{e f f}\left(q_{I C}\right) \chi^{\text {neutron }}\left(q_{I C}\right) \simeq 50 .
$$

Le système est donc très proche d'une instabilité onde de densité de spin $(\delta \rightarrow 0)$ [16-19].

Pour compléter la description du spectre des excitations magnétiques, il a fallu utiliser la technique de diffusion inélastique des neutrons polarisés, afin de pouvoir séparer de faibles signaux magnétiques du bruit de fond nucléaire $[14,20]$. À partir de l'ensemble des mesures de diffusion inélastiques de neutrons, on peut obtenir une description phénoménologique de la susceptibilité magnétique jusqu'à une énergie de l'ordre de $\sim 40 \mathrm{meV}$. Le spectre des excitations magnétiques se présente sous la forme d'un quartet d'excitations incommensurables aux vecteurs d'onde $\mathbf{q}_{I C}=( \pm 0.3, \pm 03)$, auquel s'ajoute une seconde contribution magnétique de faible intensité, s'étalant sur la quasi-totalité d'une zone de Brillouin, et centrée sur le vecteur d'onde $\mathbf{q}_{F M}=0$ (Fig. 6c). La partie imaginaire de la susceptibilité 


\begin{tabular}{cccc}
\hline $\mathrm{Sr}_{2} \mathrm{RuO}_{4}$ & & $\mathrm{~T}=1.6 \mathrm{~K}$ & $\mathrm{~T}=150 \mathrm{~K}$ \\
\hline $\operatorname{Re} \chi_{F M}$ & $=$ & $22 \pm 1 \mu_{B}^{2} \cdot e V^{-1} / R u$ & $22 \pm 2$ \\
$\Delta q_{F M}$ & $=$ & $0.53 \pm 0.04 \mathrm{r} .1 . \mathrm{u}$ & $0.47 \pm 0.06$ \\
$\Gamma q_{F M}$ & $=$ & $15 \pm 1.5 \mathrm{meV}$ & $19 \pm 3.5$ \\
\hline $\operatorname{Re} \chi_{I C}=$ & $213 \pm 10 \mu_{B}^{2} \cdot \mathrm{eV}^{-1} / R u$ & $89 \pm 7$ \\
$\Delta q_{I C}$ & $=$ & $0.126 \pm 0.006 \mathrm{r} .1 . \mathrm{u}$ & $0.20 \pm 0.06$ \\
$\Gamma q_{I C}$ & $=$ & $11.1 \pm 0.8 \mathrm{meV}$ & $17.8 \pm 2.9$ \\
\hline
\end{tabular}

prend la forme suivante :

$$
\begin{aligned}
\operatorname{Im} \chi(\mathbf{q}, \omega) & =\operatorname{Im} \chi_{F M}(\mathbf{q}, \omega)+\operatorname{Im} \chi_{I C}(\mathbf{q}, \omega) \\
\operatorname{Im} \chi_{F M}(\mathbf{q}, \omega) & =\operatorname{Re} \chi_{F M} \frac{\Gamma_{F M} \omega}{\omega^{2}+\Gamma_{F M}^{2}} \exp \left[-4 \ln \left(2 \frac{|\mathbf{q}|^{2}}{\Delta q_{F M}^{2}}\right]\right. \\
\operatorname{Im} \chi_{I C}(\mathbf{q}, \omega) & =\operatorname{Re} \chi_{I C} \frac{\Gamma_{I C} \omega}{\omega^{2}+\Gamma_{I C}^{2}} \sum_{\mathbf{q}_{I C}} \exp \left[-4 \ln (2) \frac{\left|\mathbf{q}-\mathbf{q}_{I C}\right|^{2}}{\Delta q_{I C}^{2}}\right]
\end{aligned}
$$

dont les paramètres sont donnés dans le tableau ci-joint.

\subsection{Comparaison avec d'autres techniques expérimentales}

La paramétrisation du spectre des excitations magnétiques en unité absolue permet de comparer les mesures de diffusion inélastique des neutrons avec d'autres techniques expérimentales [14]. On obtient ainsi un accord quantitatif remarquable avec les mesures de relaxation spin-réseau en RMN, qui sondent la susceptibilité magnétique locale (c'est-à-dire intégré sur $q$ ) dans la limite $\omega \rightarrow 0$. Dans ce cas on voit que des fluctuations magnétiques de faible intensité mais très étalées dans la zone de Brillouin ont une contribution équivalente à celle de fluctuations fortes mais très localisées autour de vecteurs d'onde spécifiques. La comparaison avec les mesures de chaleur spécifique indique que ce sont les contributions quasi-ferromagnétiques qui contribuent essentiellement au coefficient de sommerfeld qui donne la contribution électronique à la chaleur spécifique. Il est également possible de mener une comparaison (à ce jour encore qualitative) avec le spectre des excitations électroniques mesuré par photoémission résolue en angle.

Pour simplifier nous supposons ici que les fluctuations IC sont uniquement sur les vecteurs d'onde $q_{I C}$ et que les fluctuations quasi-ferromagnétiques sont quant à elles quasi-indépendantes de $Q$. Ces deux types d'excitations contribuent de façon différentes à l'énergie propre des quasiparticules :

$$
\begin{aligned}
& \Sigma_{I C}(k, \omega) \sim-U_{e f f}^{2} \int_{-\infty}^{\infty} \frac{d \Omega}{\pi} \operatorname{Re} \chi_{I C} \frac{\Gamma_{I C} \Omega}{\Omega^{2}+\Gamma_{I C}^{2}} \sum_{q_{i c}} \frac{n^{B}(\Omega)+n^{F}\left(\xi_{k+q_{i c}}\right)}{\omega+\Omega-\xi_{k+q_{i c}}+i \epsilon} \\
& \Sigma_{F M}(k, \omega) \sim U_{e f f}^{2} \int_{-\infty}^{\infty} \frac{d \Omega}{\pi} \operatorname{Re} \chi_{F M} \frac{\Gamma_{F M} \Omega}{\Omega^{2}+\Gamma_{F M}^{2}} \int_{-\infty}^{\infty} \frac{d v}{\pi} \rho(v) \frac{n^{B}(\Omega)+n^{F}(\nu)}{\omega+\Omega-v+i \epsilon}
\end{aligned}
$$

$\rho(v)$ représente la densité d'états électronique. Pour les fluctuations IC qui connectent principalement les bandes quasi-1D $\alpha$ et $\beta$, on s'attend à une anomalie dans la relation de dispersion de ces bandes à une énergie de l'ordre de $\Gamma_{I C}$ par rapport au niveau de Fermi comme représenté schématiquement sur la Figure 7a. Pour les fluctuations FM, potentiellement associées à la bande quasi-2D $\gamma$, on s'attend à une anomalie similaire pour une énergie de l'ordre de $\Gamma_{F M}$. La faiblesse de $\operatorname{Re} \chi_{F M}$ est alors compensé par le fait qu'on sonde un grand nombre d'états électroniques via $\rho(\nu)$. Jusqu'à présent, aucune anomalie n'a été observée sur les bandes $\alpha$ et $\beta$, par contre la bande $\gamma$ présente une anomalie à une énergie sensiblement supérieure à $\Gamma_{F M}$. Elle peut être attribuée à une interaction avec les fluctuations magnétiques. Elle est également attribuée à une interaction avec des phonons optiques. Mais cette 


\section{Collection SFN}

a)
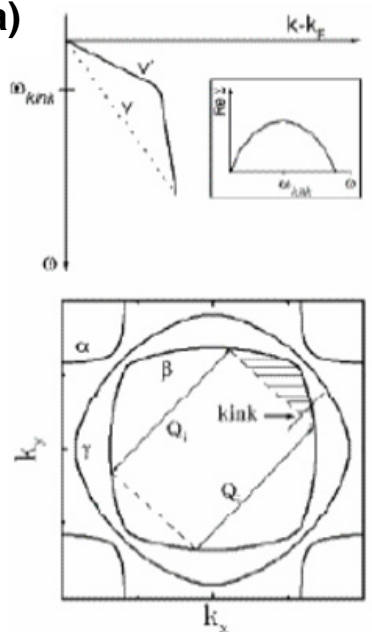

b)
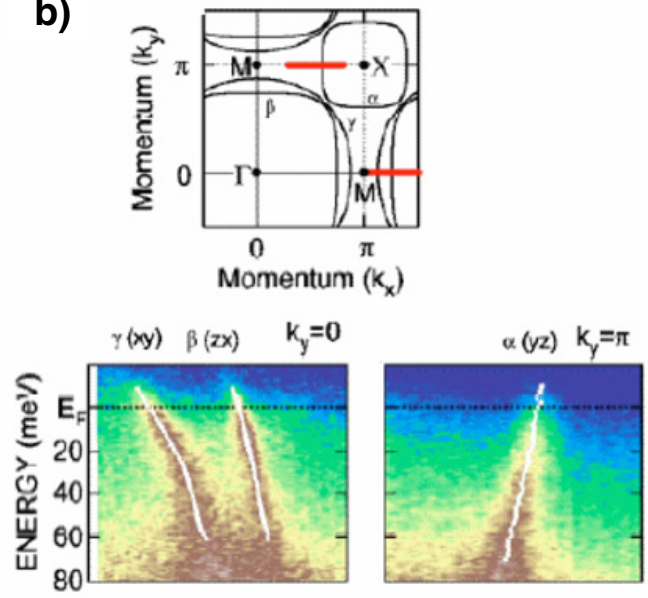

Figure 7. a) Description schématique de la relation de dispersion des quasiparticules, présentant une discontinuité (kink), à l'énergie pour laquelle la partie réelle de l'énergie propre $\operatorname{Re} \Sigma k, \omega$ présente un maximum. Un tel effet a été prédit pour la bande $\beta$ le long des directions diagonales et devrait résulter de l'interaction des quasiparticules de la bande $\beta$ avec les fluctuations incommensurables caractérisés par le vecteur d'onde incommensurable [22]. b) Mesure de la dispersion des quasiparticules pour les bandes $\alpha, \beta$ et $\gamma$ par photoémission résolue en angle [21].

interprétation est peu crédible, dans la mesure où les phonons incriminés sont beaucoup plus haut en énergie, comme montré par les mesures de diffusion inélastique des neutrons [23].

Le cas du composé $\mathrm{Sr}_{2} \mathrm{RuO}_{4}$ illustre la relation forte qui lie le spectre des quasiparticules et le spectre des excitations électroniques. Dans le cas d'un système multi-bandes, le problème peut vite devenir compliqué. Nous nous sommes limités ici à une description simplifiée dans laquelle nous ne prenons pas en considération les effets de couplage spin-orbite [24] ni le terme de couplage de Hund, qui introduisent des anisotropies dans l'espace des spins [20] et des effets de couplage inter-bandes. L'origine et le rôle des fluctuations quasi-FM restent encore mystérieux. Toutefois ces fluctuations semblent potentiellement importantes pour comprendre les propriétés électroniques. Pour comprendre d'un point de vue théorique l'origine de la supraconductivité triplet via un mécanisme d'échange de fluctuations magnétiques, il est important d'avoir une connaissance détaillé de la susceptibilité magnétique et de la structure électronique [16, 25].

- Remarque : D’un point de vue pratique, si nous prenons un même échantillon et que nous mesurons les fluctuations IC sur un spectromètre 3-axes thermique, il faut pour mesurer les fluctuations FM sur un spectromètre 3-axes thermique polarisé (avec une statistique équivalente) compter a peu près .... 1000 fois plus longtemps ! Un facteur 30 pour la perte de flux, une facteur 3 pour l'analyse de polarisation et un autre facteur 10 pour le poids relatif des fluctuations IC/FM...

\subsection{Etat supraconducteur}

\subsection{Gap de spin et excitations magnétiques résonnantes}

Nous considérons en premier le cas des nouveaux supraconducteurs à base de Fer et d'arsenic. Ces matériaux ont un fort caractère $2 \mathrm{D}$ et se caractérisent par un empilement de feuillets de FeAS. Leurs propriétés électroniques sont dominées par les 5 électrons de la couche $3 \mathrm{~d}$ du Fe. Cela donne une structure électronique complexe à 5 bandes résultant de l'hybridation des orbitales $3 \mathrm{~d}$ du Fe. Du point 
a)

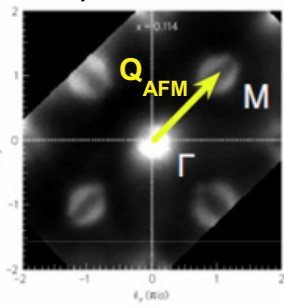

b)

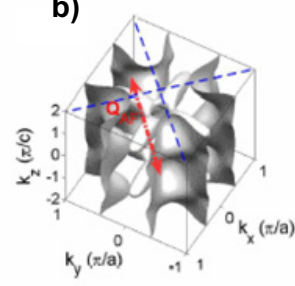

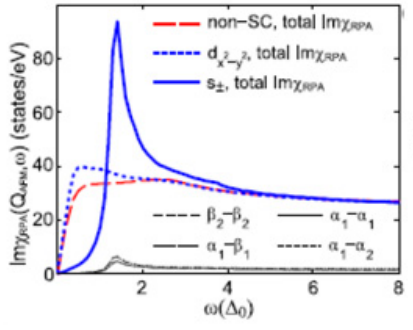

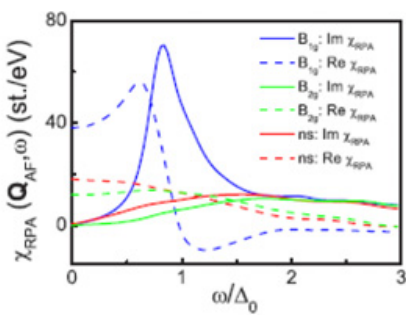

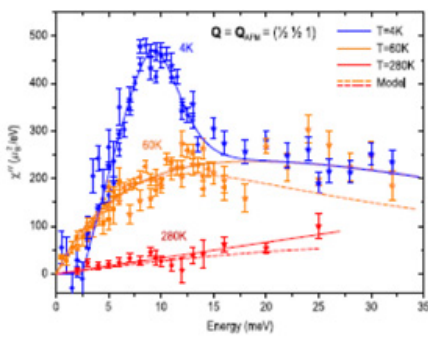

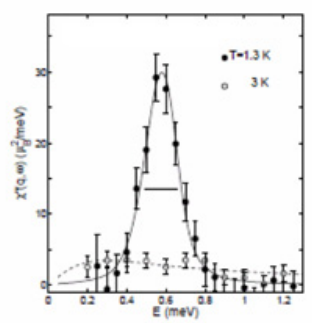

Figure 8. Structure électronique, partie imaginaire de la susceptibilité RPA et partie imaginaire de la susceptibilité magnétique mesurée par diffusion inélastique des neutrons : a) composé $\left.\mathrm{Ba}(\mathrm{Fe}, \mathrm{Co})_{2} \mathrm{As}_{2}, \mathrm{~b}\right)$ composé $\mathrm{CeCoIn}_{5}$.

de vue des propriétés magnétiques, les électrons interagissent via la répulsion coulombienne sur le site du $\mathrm{Fe}(U)$ et le couplage de Hund $(J)$ qui couple fortement les électrons sur différentes orbitales du Fe.

On peut simplifier la structure électronique en limitant à une structure électronique ne contenant que deux bandes engendrant une poche de trous au point $\Gamma\left(\xi_{k, \Gamma} \simeq E_{\Gamma}-\frac{\hbar^{2}}{2 m_{0}}\left(k_{x}^{2}+k_{y}^{2}\right)-\mu\right.$ avec $\left.E_{\Gamma}<\mu\right)$ et des poches d'électrons au point $M\left(\xi_{k, M} \simeq E_{M}+\hbar^{2}\left(\frac{\left(k_{x}+k_{y}\right)^{2}}{2 m_{1}}+\frac{\left(k_{x}-k_{y}\right)^{2}}{2 m_{2}}\right)-\mu\right.$ avec $\left.E_{M}>\mu\right)$ (Fig. 8a). Les points $\Gamma$ et $M$ sont reliés par le vecteur d'onde antiferromagnétique $\mathbf{Q}_{A F}$. A dopage nul, le système présente un ordre onde de densité de spins avec comme vecteur de propagation $\mathbf{Q}_{A F}$. Cette état magnétique résulte d'un effet d'emboitement des poches de trous et d'électrons, effet de nesting $\left(-\xi_{k+Q_{A F}, \Gamma}=\xi_{k, M}\right)$. En faisant varier la densité électronique, soit par dopage en trous, soit par dopage en électrons, la phase onde de densité de spin est détruite. Le système devient alors métallique à haute température et supraconducteur en dessous d'une température critique $\mathrm{T}_{c}$. La Figure 5a, montre l'évolution en fonction de la température du spectre des excitations magnétiques mesurés au vecteur d'onde $\mathbf{Q}_{A F}$ dans le composé supraconducteur $\mathrm{Ba}(\mathrm{Fe}, \mathrm{Co})_{2} \mathrm{As}_{2}$ [26]. Dans l'état normal, la partie imaginaire de la susceptibilité magnétique est bien décrite par la formule de OrnsteinZernike (Eq. (2.8)), tel que : $\left[\operatorname{Re} \chi\left(Q_{A} F\right)\right]^{-1} \sim \xi^{-2} \sim \omega_{s f} \sim(T+\Theta)$. Dans l'état supraconducteur, les fluctuations de basse énergie sont supprimées, indiquant l'ouverture d'un gap de spin dans le spectre des excitations magnétiques. Apparait alors, une pic à une énergie $\omega_{r}$ inférieure à $2 \Delta$, soit l'énergie qu'il faut fournir pour casser les paires de Cooper au vecteur d'onde $\mathbf{Q}_{A F}$. Ce pic est communément appelé pic de résonance magnétique. A plus haute énergie les spectres des excitations magnétiques changent peu en passant de l'état normal à l'état supraconducteur.

Le pic de résonance magnétique est une excitation spécifique à l'état supraconducteur. Son intensité et son énergie caractéristique présentent des évolutions en température similaires à celle du paramètre d'ordre supraconducteur [26]. En fonction du dopage, la température critique $\mathrm{T}_{c}$ et $\omega_{r}$ varient dans un rapport constant: $\omega_{r} \simeq 4.3 k_{B} T_{c}$. Le pic de résonance magnétique est décrit comme un exciton de spin, c'est-à-dire un état lié électron-trou poussé en dessous du continuum de Stoner par les corrélations 


\section{Collection SFN}

électroniques. La Figure 5a présente un calcul RPA de la susceptibilité magnétique dans l'état normal et dans l'état supraconducteur [27]. Dans l'état supraconducteur, deux symétries sont considérées pour le gap supraconducteur: (i) la symétrie $d$ avec $\Delta_{k}=\frac{\Delta}{2}\left(\cos \left(k_{x}\right)-\cos \left(k_{y}\right)\right)$, (ii) la symétrie $s_{ \pm}$pour laquelle $\Delta$ est isotrope, mais de signes opposés pour les poches de trous et d'électrons. Le calcul montre qu'un exciton de spin n'apparait que dans le cas de la symétrie $s_{ \pm}$. A partir de l'observation d'un pic de résonance magnétique, il est donc possible d'obtenir des informations sur les propriétés de symétrie du paramètre d'ordre supraconducteur.

Les composés supraconducteurs à base de FeAS sont des composés métalliques dans lesquels les effets de corrélations sont importants, mais suffisamment modérés, pour que dans l'état normal ils conservent les propriétés d'un liquide de Fermi. D'autres supraconducteurs non conventionnels ont un état normal plus pathologique. C'est le cas par exemple des systèmes de fermions lourds. L'image du liquide de Fermi demeure qualitativement valable, mais la masse effective des quasiparticules présentes au voisinage du niveau de Fermi peut atteindre jusqu'à 1000 fois celle d'un électron libre. Nous considérons à présent le cas du composé à fermions lourds supraconducteur $\mathrm{CeCoIn}_{5}$.

La structure électronique du composé $\mathrm{CeCoIn}_{5}$ est difficile à décrire, dans la mesure où elle fait intervenir plusieurs bandes d'électrons $f$ et d'électrons de conduction hybridées de façon complexe. On peut simplifier le problème en se ramenant à un problème à deux bandes résultant de l'hyridation d'une bande $f$ et d'une bande d'électrons de conductions $c$, tel que $\xi_{k, \pm}=\frac{1}{2}\left[\left(\xi_{f}+\xi_{c}\right) \pm\right.$ $\sqrt{\left.\left(\xi_{f}-\xi_{c}\right)-4 V_{f c}^{2}\right]}$, où $V_{f c}$ est le potentiel d'hybridation (Fig. 8b). La surface de Fermi présente des feuillets ayant des propriétés de nesting pour le vecteur d'onde $\mathbf{Q}_{A F}$ (Fig. 5b). Les mesures de diffusion inélastique des neutrons au vecteur d'onde $\mathbf{Q}_{A F}$ montrent l'apparition d'un pic de résonance magnétique très intense dans l'état supraconducteur [28]. Dans une approche RPA, cette excitation peut être interprétée comme un exciton de spin. Les calculs de la susceptibilité RPA indiquent qu'une telle excitation est compatible avec une supraconductivité $d$ qui présente des lignes de noeuds le long des directions (100) et (010), tel que $\Delta_{k}=\frac{\Delta}{2}\left(\cos \left(k_{x}\right)-\cos \left(k_{y}\right)\right)$ (symétrie $\left.B_{1 g}\right)$. Par contre, l'exciton de spin est absent pour une supraconductivité de symétrie $d$ avec des lignes de noeuds tournés à $45^{\circ}$ (symétrie $B_{2 g}, \Delta_{k}=\Delta \sin \left(\frac{k_{x}}{2}\right) \sin \left(\frac{k_{y}}{2}\right)[27]$.

\subsection{Excitations magnétiques résonantes et excitons de spin}

Un grand nombre de supraconducteurs non conventionnels $(S=0)$ présentent des pics de résonance magnétique au voisinage du vecteur d'onde antiferromagnétique. Ces systèmes sont variés : on trouve les oxydes de cuivre supraconducteurs à haute température critique, les supraconducteurs à base de Fer, des supraconducteurs à fermions lourds [30]. Dans une approche de magnétisme itinérant, l'apparition de pic de résonance magnétique est lié au changement de la susceptibilité magnétique sans interaction dans l'état supraconducteur. Dans l'état supraconducteur, les fluctuations de basse énergie de l'état normal sont supprimées et transférées au niveau de la bordure inférieure du continuum de Stoner. Lorsque $\operatorname{Im} \chi^{o}$ se présente sous la forme d'une fonction créneau en entrant dans le continuum de Stoner à $\omega_{c}(\sim 2 \Delta)$, $R e \chi^{o}$ obtenue par transformation de Karmers-Kronig présente automatiquement une singularité à $\omega_{c}$ (Fig. 9). Nous pouvons alors envisager deux cas de figure.

- L'approche itinérante pure : la condition $\operatorname{Re} \chi^{o}\left(q, \omega_{r}\right)=1 / U$ avec $\left(\omega_{r}<\omega_{c}\right)$ caractérise l'apparition d'un excition de spin (Fig. 9a). Un mode collectif se forme en dessous du continum de Stoner dans la susceptibilité RPA (Fig. 9c, courbe rouge).

- L'approche localisée hybride : ici nous considérons des excitations magnétiques localisées caractérisées par une relation de dispersion $\Omega_{q}$. Ces excitations sont couplées au bain d'électrons 

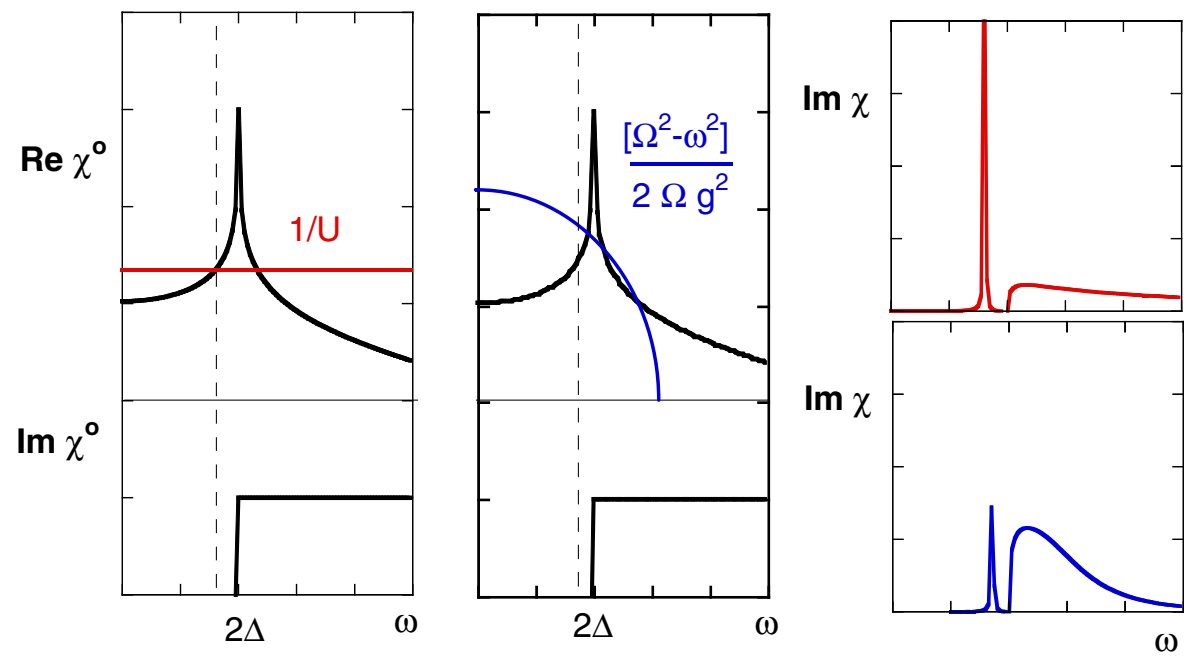

Figure 9. Dépendances en énergie de la partie rélle, $R e \chi^{\circ}$ et de la partie imaginaire de la susceptibilité magnétique, $\operatorname{Im} \chi^{o}:$ a) condition de pôles dans un cas itinérant pur (ligne rouge), b) condition de pôle dans un cas mixte itinérantlocalisé (ligne bleue). c) Partie imaginaire de la susceptibilité RPA pour les cas représentés en a) et b).

de conduction. En accord avec l'Eq. (2.15), la condition $\operatorname{Re} \chi^{o}(q, \omega)=\frac{\left[\Omega_{q}^{2}-\omega^{2}\right]}{2 \Omega_{q} g^{2}}$ donne les pôles de la susceptibilité en interaction (Fig. 9b). Si $\Omega_{q}<\omega_{c}$, les modes localisés ne sont plus amortis dans l'état supraconducteur et leur intensité augmente. Si $\Omega_{q}>\omega_{c}$, les modes localisés demeurent dans le continuum de Stoner et restent amortis comme dans l'état normal. De plus, pour $\omega<\Omega_{q}$, la condition de pôles prend la forme : $\operatorname{Re} \chi^{o}(q, \omega) \simeq \frac{\Omega_{q}}{2 g^{2}}=1 / U_{e f f}$. A l'excitation localisée amortie à haute énergie vient s'ajouter un exciton de spin à plus basse énergie (Fig. 9 c, courbe bleue). Lorsque $\Omega_{q} \simeq \omega_{c}$, la notion d'excitation localisée pure et d'excitation collective de type électron-trou pure est perdue et ces deux types d'excitations s'hybrident fortement, donnant lieu à des modes mixtes.

La première interprétation est celle qui est le plus largement retenue pour expliquer la formation d'un pic de résonance magnétique. La seconde est par exemple utilisée pour interpréter la présence d'un pic de résonance magnétique dans le composé $\mathrm{UPd}_{2} \mathrm{Al}_{3}$ [31-33]. Ce composé à fermions lourds est caractérisé par la présence de deux types d'électrons $f$ : les uns itinérants et les autres localisés induisant la réponse magnétique localisée.

Il est important de noter que des excitations magnétiques résonantes sont le plus souvent observés dans des supraconducteurs non conventionnels, mais il existe également des cas où des excitations magnétiques similaires sont observées dans des matériaux non supraconducteurs, présentant toutefois un autre type d'instabilité électronique qui ouvre un gap dans le spectre d'excitation des charges. C'est le cas du système isolant-Kondo $\mathrm{YB}_{12}[34,35]$ et du composé antiferro-quadrupolaire $\mathrm{CeB}_{6}[36,37]$.

\subsection{Propriétés électroniques : effet de feedback}

Nous considérons ici le cas des oxydes de cuivre supraconducteurs à haute température. Ces matériaux ont une structure de type perovskite en couche caractérisée par un empilement de plans carrés $\mathrm{CuO}_{2}$. A dopage nul, ces matériaux sont des isolants de Mott antiferromagnétiques. Les spins $S=1 / 2$ du cuivre sont alors localisés. En faisant varier la densité électronique par dopage, l'ordre antiferromagnétique est rapidement détruit et le système devient métallique et supraconducteur en dessous de la température critique $\mathrm{T}_{c}$. L'état supraconducteur est de symétrie $d$ et singlet de spin. A faible dopage, ces 


\section{Collection SFN}

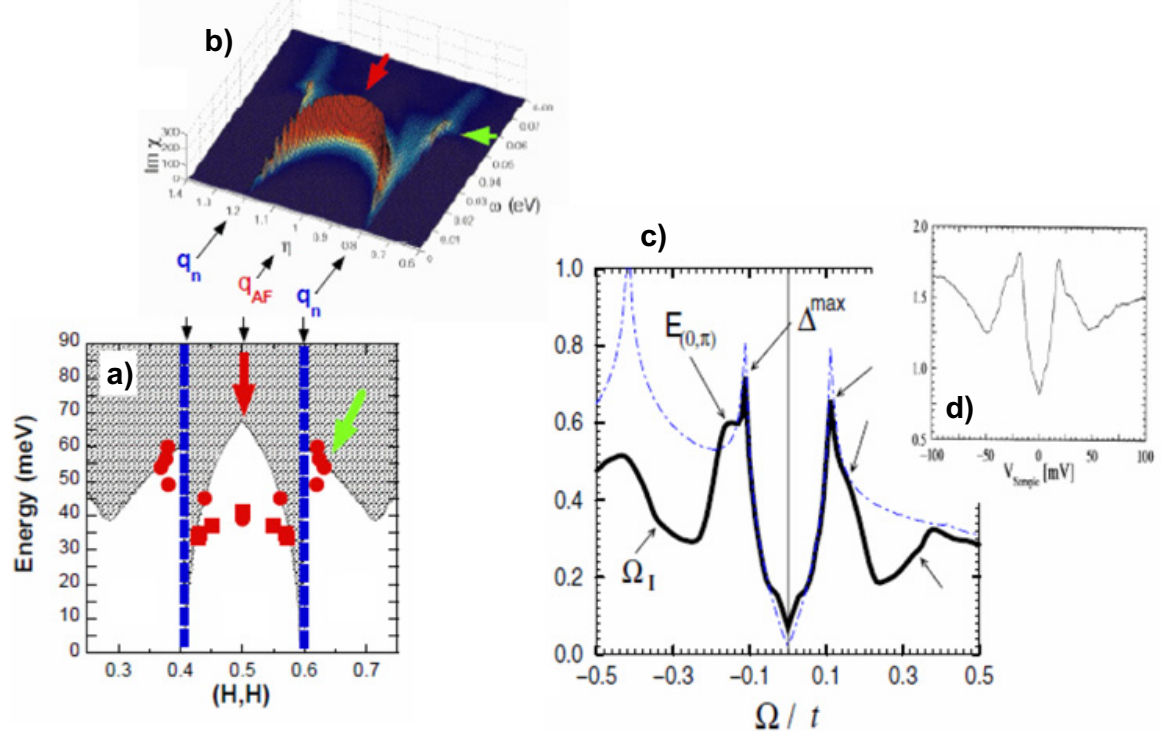

Figure 10. a) Dispersion des excitations magnétiques résonnantes dans $\mathrm{YBa}_{2} \mathrm{Cu}_{3} \mathrm{O}_{6.85}$. La zone grise représente le continuum de Stoner tel que déduit des mesures de photoémission résolue en angle. b) Partie imaginaire de la susceptibilité magnétique calculée dans une approche RPA [44]. c) Calcul de la densité d'états électronique [11]. Insert : densité d'états électroniques mesurée par effet tunnel.

composés sont de mauvais métaux et leurs propriétés électroniques ne peuvent pas être décrites par un modèle de liquide de Fermi. En augmentant le dopage, un comportement de métal plus standard est progressivement restauré. On peut donc décrire les propriétés magnétiques de ces matériaux soit en partant d'un isolant de Mott dopé (approche localisée), soit en partant d'un liquide de Fermi et en diminuant le dopage (approche itinérante). C'est cette dernière approche que nous adoptons ici. C'est dans le composé dopé en trous $\mathrm{YBa}_{2} \mathrm{Cu}_{3} \mathrm{O}_{6+x}$ qu'un pic de résonance magnétique a été pour la première fois observé en 1991 par J. Rossat-Mignot et al. [38]. L'observation d'une telle excitation a par la suite été généralisée à d'autres familles d'oxydes de cuivre supraconducteurs telles que $\mathrm{Bi}_{2} \mathrm{Sr}_{2} \mathrm{CaCu}_{2} \mathrm{O}_{8+\delta}$ [39] et $\mathrm{Tl}_{2} \mathrm{Ba}_{2} \mathrm{CuO}_{6+\delta}$ [40]. Les excitations résonantes sont à présent observées dans la quasi-totalité des oxydes de cuivre supraconducteurs étudiés par diffusion inélastique des neutrons.

Le pic de résonance magnétique est maximum au vecteur d'onde $\mathbf{Q}_{A F}$ et a une énergie $\omega_{r}$. Autour de ce vecteur d'onde il présente une dispersion incurvée vers le bas [41]. Cette dispersion s'explique particulièrement bien dans un modèle d'exciton de spin [42]. En effet le long la direction (110), la bordure inférieure du continuum de Stoner est maximum à $\mathbf{Q}_{A F}$ et s'annule en $\mathbf{Q}_{N}$ qui est le vecteur d'onde qui relie les noeuds du gap supraconducteur. L'exciton de spin adopte une dispersion qui reproduit la forme de la bordure du continuum de Stoner. En s'écartant de $\mathbf{Q}_{A F}$, l'exciton de spin disperse tout d'abord vers le bas et disparait en entrant dans le contiuum de Stoner. Puis à plus grand vecteur d'onde, il réapparait à plus haute énergie, avec une intensité fortement réduite [43]. La dispersion des excitations résonantes observées expérimentalement (Fig. 10a) est bien reproduite par la susceptibilité RPA calculée [44].

L'apparition des modes résonants s'accompagne de nombreuses anomalies dans le spectre des quasiparticules mesuré par photo-émission résolue en angle $(A(k, \omega))$ et dans la densité d'états électroniques mesuré par effet tunnel $\left(\rho(\omega)=\frac{1}{N} \sum_{k} A(k, \omega)\right)$ [45]. Tout comme les modes résonants, ces anomalies dans les mesures de spectroscopie de charge disparaissent en passant dans l'état normal. 
A partir des mesures de diffusion inélastique de neutrons, il est possible de modéliser la dispersion des modes résonants et de les incorporer dans les équations auto-cohérentes qui donnent $\Sigma(k, \omega)$ et $\Phi(k, \omega)$ dans l'état supraconducteur (Eq. (3.26)). La Figure 10c montre le calcul de $\rho(\omega)$ ainsi obtenu [11]. La courbe en pointillés bleus montre la densité d'états électroniques pour $\Sigma(k, \omega)=0$ et $\Phi(k, \omega)=\Delta_{k}=\frac{\Delta_{o}}{2}\left(\cos \left(k_{x}\right)-\cos \left(k_{y}\right)\right)$. On voit apparaitre d'importantes différences entre les deux courbes, dont la formation d'une vallée à une énergie de l'ordre $\Omega_{1}=\Delta_{o}+\omega_{r}$. En prenant en compte les modes résonants, le calcul reproduit la structure de la densité d'états électroniques mesurée par effet tunnel (Fig. 10c - insert).

Des calculs similaires ont été réalisés dans le composé $\mathrm{UPd}_{2} \mathrm{Al}_{3}$ [47], reliant l'observation d'un pic de résonance magnétique mesuré par diffusion de neutrons [31] aux anomalies dans $\rho(\omega)$ mesurés par effet tunnel [46]. Dans le plomb, qui est un supraconducteur conventionnel, les anomalies dans $\rho(\omega)$ sont directement reliés à la structure de la densité d'états de phonons mesuré par diffusion inélastique des neutrons [48].

Nous avons vu ici que les propriétés électroniques conditionnaient le spectre des excitations magnétiques (restructuration du continuum de Stoner, apparition de modes collectifs dispersifs). De même, le changement du spectre des excitations magnétiques induit une modification du spectre électronique. C'est ce que l'on appelle l'effet de feedback.

\section{CONCLUSION}

Nous avons essayé de montrer comment dans des métaux et des supraconducteurs (non conventionnels) les propriétés des charges et les propriétés magnétiques étaient couplées au travers de la susceptibilité de spin. Celle-ci peut être mesurée directement par diffusion inélastique des neutrons et calculée à partir d'un modèle de bandes électroniques. Dans les supraconducteurs non conventionnels, la mesure de la susceptibilité magnétique renseigne sur la symétrie du paramètre d'ordre supraconducteur, l'amplitude des corrélations électroniques et peut être injectée dans des calculs auto-cohérents permettant d'évaluer le rôle des fluctuations magnétiques dans le mécanisme d'appariement supraconducteur.

Nous avons considéré ici une approche basée sur un magnétisme de bandes qui implique qu'une description en terme de liquide de Fermi de l'état métallique soit valable et les corrélations électroniques modérées. Lorsque les corrélations électroniques deviennent fortes et de l'ordre de la largeur de bande, la description des propriétés électroniques et magnétiques que nous avons présenté doit être prise avec beaucoup de prudence et demeure indicative. La plupart des supraconducteurs non conventionnels sont fortement corrélés et/ou proches d'une instabilité magnétique. Ils développent de fait des propriétés dites non-liquide de Fermi.

Nous avons également limité la description expérimentale au cas de systèmes non perturbés. Les perturbations couramment utilisées pour les supraconducteurs non conventionnels sont de deux types : (i) locales ou (ii) uniformes. Dans le premier cas, il s'agit en général d'introduire des impuretés non magnétiques qui sont connues pour réduire la supraconductivité non conventionnelle et d'étudier leur effet sur la dynamique de spins $[49,50]$. Dans le second cas, on peut par exemple appliquer un champ magnétique dans l'état supraconducteur. Il faut alors prendre en compte l'effet orbital qui est lié à l'action d'un champ magnétique sur les particules chargés et l'effet Zeeman qui est lié au couplage du champ magnétique avec le spin des électrons [51-54].

Nous nous sommes également restreints (en général) au cas d'un état supraconducteur pur. Nous avons ainsi évité de traiter le cas de la coexistence de l'état supraconducteur avec un état magnétiquement ordonné. Or ce cas de figure est fréquemment rencontré dans les diagrammes de phase des supraconducteurs non conventionnels. Dans ce cas le spectre des excitations magnétiques peut être très complexe, mélangeant différents types d'excitations magnétiques [55]. Par ailleurs, lorsqu'un état supraconducteur de symétrie $d$ coexiste avec un état onde de densité de spin antiferromagnétique, un autre état de paires d'électrons apparait théoriquement comme la combinaison des deux premiers : les 


\section{Collection SFN}

paires d'électrons ont alors une quantité de mouvement $\mathbf{Q}_{A F}$, un état de spin triplet et une symétrie $d$. Si ces paires $\pi$-triplet existent, elles contribuent à la susceptibilité de spin [56] et sont donc détectables par diffusion des neutrons.

Le fait que de nombreux supraconducteurs apparaissent au voisinage d'une phase magnétiquement ordonnée, suggère que les fluctuations magnétiques jouent un rôle dans le mécanisme d'appariement supraconducteur. Toutefois, l'idée peut être étendue à un autre état ordonné dont les fortes fluctuations associées au paramètre d'ordre joueraient un rôle dans le mécanisme d'appariement supraconducteur. Citons par exemple, les états électroniques caractérisés par une densité de charge uniforme, mais présentant des boucles de courants. Ces phases génèrent une réponse magnétique de type orbitale qui est détectable par diffusion des neutrons [57, 58].

$\mathrm{Au}$ fil des découvertes de nouveaux supraconducteurs non conventionnels et de l'émergence de nouveaux concepts théoriques pour en comprendre la nature, la diffusion des neutrons a joué un rôle croissant et fourni des résultats majeurs.... tout du moins à chaque fois qu'il est possible de disposer d'échantillons (monocristallins) d'un volume suffisant.

\section{Références}

[1] S.W. Lovesey, Theory of Neutron Scattering from Condensed matter, Vol. 2, Clarendon, Oxford (1984).

[2] N. Pottier, Physique statistique hors d'équilibre : Processus irréversibles linéaires, EDP Sciences (2007).

[3] Levy, Magnétisme et Supraconductivité, EDP Sciences (1997).

[4] T. Moriya, Spin fluctuations in itinerant electron magnetism, Solid-State Sciences 56, Springer Verlag, Berlin Heidelberg (1985).

[5] H. Bruus \& K. Flensberg, Many Body Quantum theory in Condensed matter: an introduction, Oxford University Press (2004).

[6] P. Monthoux, D. Pines \& G.G. Lonzarich, Nature 450, 1177 (2007).

[7] F. Onufrieva \& P. Pfeuty, Phys. Rev. Lett. 82, 3136 (1999).

[8] A. Damascelli et al., Rev. Mod. Phys. 75, 473 (2003).

[9] S. Pailhés, Thèse, Université Paris XI (2004).

[10] R. Joynt \& T.M. Rice, Phys. Rev. B 38, 2345 (1988).

[11] F. Onufrieva \& P. Pfeuty, Phys. Rev. Lett. 102, 207003 (2009).

[12] A. Mackenzie \& Y. Maeno, Rev. Mod. Phys., 75, 658 (2003).

[13] A. Damascelli, D.H. Lu, K.M. Shen, N.P. Armitage, F. Ronning, D.L. Feng, C. Kim, Z.-X. Shen, T. Kimura, Y. Tokura, Z.Q. Mao, \& Y. Maeno, Phys. Rev. Lett. 85, 5194 (2000).

[14] P. Steffens, Thèse, Université de Cologne (2007).

[15] I.I. Mazin \& D.I. Singh, Phys. Rev. Lett 79, 733 (1997).

[16] I.I. Mazin \& D.I. Singh, Phys. Rev. Lett 82, 4324 (1999).

[17] Y. Sidis, M. Braden, P. Bourges, B. Hennion, S. NishiZaki, Y. Maeno, \& Y. Mori, Phys. Rev. Lett. 83, 3320 (1999).

[18] M. Braden, Y. Sidis, P. Bourges, P. Pfeuty, J. Kulda, Z. Mao, \& Y. Maeno, Phys. Rev. B 66, 064522 (2002).

[19] M. Braden, P. Steffens, Y. Sidis, J. Kulda, P. Bourges, S. Hayden, N. Kikugawa \& Y. maeno, Phys. Rev. Lett. 92, 097402 (2002).

[20] P. Steffens, Y. Sidis, P. Link, K. Schmalzl, S. Nakatsuji, Y. Maeno \& M. Braden, Phys. Rev. Lett. 99, 217402 (2007).

[21] H. Iwasawa, Y. Aiura, T. Saitoh, I. Hase, S.I. Ikeda, Y. Yoshida, H. Bando, M. Higashiguchi, Y. Miura, X.Y. Cui, K. Shimada, H. Namatame, \& M. Taniguchi, Phys. Rev. B 72, 104514 (2005). 
[22] D. Manske, I. Eremin, \& K.H. Bennemann, Phys. Rev. B 67, 134520 (2003).

[23] M. Braden, W. Reichardt, Y. Sidis, Z. Mao, \& Y. Maeno, Phys. Rev. B 76, 014505 (2007).

[24] I. Eremin, D. Manske, C. Joas \& K.H. Bennemann, Europhys. Lett. 58, 871 (2002).

[25] I. Eremin, D. Manske, \& K.H. Bennemann, Phys. Rev. B 65, 220502(R) (2002); I. Eremin, D. Manske, S.G. Ovchinnikov \& J.F. Annett, Annalen der Physik 13, 149 (2004).

[26] D.S. Inosov, J.T. Park, P. Bourges, D.L. Sun, Y. Sidis, A. Schneidewind, K. Hradil, D. Haug, C.T. Lin, B. Keimer \& V. Hinkov. Nature Physics 6 (2010).

[27] M.M. Korshunov \& I. Eremin, Phys. Rev. B 78, 140509 (2008).

[28] C. Stock, C. Broholm, J. Hudis, H.J. Kang, and C. Petrovic Phys. Rev. Lett. 100, 087001 (2008).

[29] I. Eremin, G. Zwicknagl, P. Thalmeier \& P. Fulde, Phys. Rev. Lett. 101, 187001 (2008).

[30] G. Yu, Y. Li, E.M. Motoyama, M. Greven, Nature Physics 5, 873 (2009).

[31] N. Bernhoeft, N. Sato, B. Roessli, N. Aso, A. Hiess, G.H. Lander, Y. Endoh, \& T. Komatsubara, Phys. Rev. Lett. 81, 4244 (1998).

[32] E. Blackburn, A. Hiess, N. Bernhoeft, and G.H. Lander Phys. Rev. B 74, 024406 (2006).

[33] Jun Chang, I. Eremin, P. Thalmeier \& P. Fulde, Phys. Rev. B 75, 024503 (2007).

[34] J.-M. Mignot, P.A. Alekseev, K.S. Nemkovski, L.-P. Regnault, F. Iga, and T. Takabatake, Phys. Rev. Lett. 94, 247204 (2005); K.S. Nemkovski, J.-M. Mignot, P.A. Alekseev, A.S. Ivanov, E.V. Nefeodova, A.V. Rybina, L.-P. Regnault, F. Iga, and T. Takabatake, Phys. Rev. Lett. 99, 137204 (2007).

[35] P.S. Riseborough, J. Magn. Magn. Mater. 226-230, 127(2001).

[36] G. Friemel, Yuan Li, A.V. Dukhnenko, N.Y. Shitsevalova, N.E. Sluchanko, A. Ivanov,V.B. Filipov, B. Keimer \& D.S. Inosov, Nature 3, 830 (2012).

[37] A. Akbari \& P. Thalmeier, Phys. Rev. Lett. 108, 146403 (2012).

[38] J. Rossat-Mignod, L.P. Regnault, C. Vettier, Ph. Bourges , P. Burlet, J. Bossy, J.Y. Henry, G. Lapertot, Physica C 185, 86 (1991).

[39] H.F. Fong, Ph. Bourges, Y. Sidis, L.P. Regnault, A.S. Ivanov, G.D. Gu, N. Koshizuka \& B. Keimer, Nature 398, 588 (1999).

[40] H.F. He, Ph. Bourges, Y. Sidis, C. Ulrich, L.P. Regnault, S. Pailhés,, N.S. Berzigiarova, N.N. Kolesnikov \& B. Keimer, Science, 295, 1045 (2002).

[41] Ph. Bourges, Y. Sidis, H.F. Fong, L.P. Regnault, J. Bossy, A.S. Ivanov, B. Keimer, Science 288 1234 (2000).

[42] F. Onufrieva and P. Pfeuty, Phys. Rev. B 65, 054515 (2002).

[43] S. Pailhés, Y. Sidis, P. Bourges, V. Hinkov, A. Ivanov, C. Ulrich, L. P. Regnault \& B. Keimer, Phys. Rev. Lett. 93, 167001 (2004).

[44] I. Eremin, D.K. Morr, A.V. Chubukov, K.H. Bennemann \& M.R. Norman Phys. Rev. Lett. 94, 147001 (2005).

[45] M. Eschrig, Adv. Phys. 55, 47 (2006).

[46] M. Jourdan, M. Huth \& H. Adrian, Nature 398, 47 (1999).

[47] N.K. Sato, N. Aso, K. Miyake, R. Shiina, P. Thalmeier, G. Varelogiannis, C. Geibel, F. Steglich, P. Fulde \& T. Komatsubara, Nature 410, 340 (2001).

[48] W.L. McMillan \& J.M. Rowell, Phys. Rev. Lett. 14108 (1965).

[49] A. Suchaneck V. Hinkov, D. Haug, L. Schulz, C. Bernhard, A. Ivanov, K. Hradil, C.T. Lin, P. Bourges, B. Keimer \& Y. Sidis, Phys. Rev. Lett. 105, 037207 (2010).

[50] Brian M. Andersen, Siegfried Graser, P.J. Hirschfeld, Europhys. Lett. 97, 47002 (2012).

[51] C. Stock, C. Broholm, Y. Zhao, F. Demmel, H.J. Kang, K.C. Rule, and C. Petrovic, Phys. Rev. Lett. 109, 167207 (2012).

[52] S. Raymond, K. Kaneko, A. Hiess, P. Steffens \& G. Lapertot Phys. Rev. Lett. 109, 237210 (2012). 


\section{Collection SFN}

[53] V.P. Michal \& V.P. Minev, Phys. Rev. B 84, 052508 (2011).

[54] A. Akbari \& P. Thalmeier, Phys. Rev. B 86, 134516 (2012).

[55] W. Rowe, J. Knolle, I. Eremin \& P.J. Hirschfeld, Phys. Rev. B 86, 134513 (2012).

[56] Zhihao Hao \& A.V. Chubukov, Phys. Rev. B 79, 224513 (2009).

[57] P. Bourges \& Y. Sidis, Comptes Rendus Physique 12, 461 (2011).

[58] C.M. Varma, Nature 468, 184 (2010). 